\title{
EXISTENCE, UNIQUENESS AND ASYMPTOTIC ANALYSIS OF OPTIMAL CONTROL PROBLEMS FOR A MODEL OF GROUNDWATER POLLUTION
}

\author{
Emmanuelle Augeraud-VÉron ${ }^{1}$, Catherine Choquet $^{1}$ and Éloïse Comte $^{1}$
}

\begin{abstract}
An optimal control problem of contaminated underground water is considered. The spatiotemporal objective takes into account the economic trade off between the pollutant use -for instance fertilizer- and the cleaning costs. It is constrained by a hydrogeological model for the spread of the pollution in the aquifer. We consider a broad range of reaction kinetics. The aim of the paper is two-fold. On the one hand, we rigorously derive, by asymptotic analysis, the effective optimal control problem for contaminant species that are slightly concentrated in the aquifer. On the other hand, the mathematical analysis of the optimal control problems is performed and we prove in particular that the latter effective problem is well-posed. Furthermore, a stability property of the optimal control process is provided: any optimal solution of the properly scaled problem tends to the optimal solution of the effective problem as the characteristic pollutant concentration decreases.
\end{abstract}

Résumé. On considère un problème de contrôle optimal de contamination des eaux souterraines. L'objectif économique prend en compte le nécessaire compromis entre l'utilisation du polluant -par exemple de l'engrais- et les coûts de dépollution. Il est soumis à la contrainte d'un modèle hydrogéologique pour la propagation de la pollution dans l'aquifère. On considère une large gamme de réactions cinétiques. L'objectif de cet article est double. D'une part, nous construisons rigoureusement, par analyse asymptotique, le problème de contrôle optimal effectif pour des contaminants présents en faible concentration dans l'aquifère. D'autre part, nous analysons le problème de contrôle optimal et nous montrons en particulier que le problème effectif est bien posé. De plus, nous démontrons une propriété de stabilité du processus de contrôle optimal : toute solution optimale du problème adimensionné converge vers la solution optimale du problème effectif lorsque l'ordre de grandeur de la concentration du polluant décroît.

1991 Mathematics Subject Classification. 49A20,49A50, 37N40, 76R99, 37N35.

April 7, 2018.

\section{INTRODUCTION}

Groundwater quality is a major concern for water supply. Human activities lead to some inevitable pollution. For instance, agriculture is the main pollutant source, with 50 to $80 \%$ of the total nitrogen and phosphorus loaded in fresh water in Europe (Lankoski and Ollikainen [17]). When pollution is linked with an economical activity, the corresponding benefits have to balance somehow the cleaning costs. This question is addressed in the present paper through an optimal control formulation. The problem is constrained by the dynamics of the pollutant in the aquifer, thus by the classical conservation principles, in the form of a highly coupled system of nonlinear partial differential equations. More precisely, the species displacement is ruled by a parabolic equation

Keywords and phrases: Optimal control problem, Hydrogeological state equations, Nonlinearly coupled problem, Parabolic and elliptic PDEs, Asymptotic analysis, Well-posedness

${ }^{1}$ University of La Rochelle, Laboratoire MIA, 23 Avenue A. Einstein, BP 33060, 17031 La Rochelle France 
of convection-diffusion-reaction type which is nonlinearly coupled to an elliptic equation ruling the velocity of the solute.

Let us quote some references devoted to optimal control problems constrained by a reaction diffusion system. The spatial dimension of the economic problem is often ignored. This is for instance the case in Van der Ploeg and de Zeeuw [20], where water production wells and pollution emission are located at the same place. Such an assumption is refined in [5] where Bordenave et al assume a time delay between the pollutant emission and the water contamination. Then, it was proved by Augeraud and Leandri [2] that optimal path may be cyclic for some specific time delay. The spatial dimension is taken into account, especially the diffusion of pollution, in Brock and Xepapadeas [6] who study a pattern formation in managed shallow lake. We also mention Camacho and Pérez-Barahona [7] who worked on a problem of land use under air pollution stress and De Frutos and Martín-Herrán [11], who consider a groundwater pollution in a multiregional framework. However, except in De Frutos and Martín-Herrán [11] and in Yuan et al [26] for numerical experiments, or in Benosman et al [4] for more theoretical results, where two-dimensional problems are considered, space dimension is often one and fluid velocity and diffusion coefficients are always assumed given and constant.

In the present paper instead, a complete hydrogeological model for the spread of the pollutant in the threedimensional aquifer is included. The velocity of the flow is given by an elliptic PDE expressing the total mass and moment conservations. Each species concentration is ruled by a parabolic PDE, of convectiondiffusion-reaction type, modeling its mass conservation. The microscopic velocity heterogeneity due to the porous medium geometry distorts the classical diffusion term, which involves actually a dispersion operator nonlinearly depending on the velocity. Reaction terms are assumed very generic, allowing all the classical (nonlinear) isotherms. The economic objective, possibly set on an infinite time length, takes into account the long-run consequences of the optimal policy. A first analysis of an optimization problem constrained by the same type of PDEs problem was performed in [1] where we obtained an existence result for a global optimal control. This result is also established here, with a new proof, and extended in two directions. On the one hand, we study the behavior of the problem for small pollutant concentrations. More precisely, we rigorously derive, by asymptotic analysis, the effective optimal control problem for contaminant species that are slightly concentrated in the aquifer. This dimensionless problem is more tractable since a part of the heterogeneity in the parameters of the original problem has disappeared thanks to the asymptotic process. The convergence result allows to substitute this new model to the original one, provided its validity range, specified by the scaling analysis, is respected. On the other hand, a mathematical analysis of the optimal control problem is performed, where we prove in particular that the latter effective problem is well-posed. The uniqueness of the optimal solution is a key result, giving sense to further studies, numerical studies for instance, and to the use of the results for decision aids. The uniqueness is proved here when assuming small pollutant concentrations but remains an open question in the general setting. Furthermore, a stability property of the optimal control process is provided: any optimal solution of the generic problem tends to the optimal solution of the effective problem as the characteristic pollutant concentration decreases. Let us recall that such a result is not straightforward. Perturbations of optimal control problems of PDEs have been studied for instance by Haraux and Murat [14] who proved in particular that the solution of the perturbed problem does not necessary converge to the one of the limit problem. Other counterexamples are provided in Kogut and Leugering [15].

The paper is organized as follows: in Section 2, we present the optimal control problem constrained by the transport and the displacement of the pollutant in groundwater. An appropriate scaling is introduced in the dimensionless form of the model for emphasizing the order of the pollutant concentrations. The main results of the paper are then claimed. Section 3 is devoted to a preliminary stability analysis of the state problem. In Section 4, a generic global existence result for the optimal control problem is proved. A well-posedness result is also achieved. The asymptotic analysis of the model for small characteristic concentrations let us derive in Section 5 the corresponding effective model, which turns out to be well-posed. 


\section{Description of the Problem}

Let us begin by the state system, which is issued from the space-time dynamics of the pollutant in the underground. We refer to [1] for a more precise derivation. Assume for the sake of simplicity that there is only one pollutant of interest. We denote by $c$ its concentration. The behavior of the pollutant is driven by the following system of PDEs

$$
\begin{aligned}
& R \psi \partial_{t} c+v \cdot \nabla c-\operatorname{div}(\psi S(v) \nabla c)=-\tilde{r}(c)+p(1-c)-g c \\
& \operatorname{div}(v)=p+g, \quad v=-\kappa \nabla \phi
\end{aligned}
$$

where the velocity of the mixture, $v$, is expressed as a function of the hydraulic head $\phi$ through the Darcy law. Here, $p$ stands for the pollutant load, that is the unknown of the upcoming optimal control problem. Term $g$ corresponds to another pollution source term which takes into account the contribution from the soil itself and other inputs. The structure of the soil is described in the porosity function, $\psi$, and in the fluid mobility tensor, $\kappa$, rating the permeability of the underground with the viscosity of the fluid. The dispersion tensor is $S(v)$. Following Scheidegger [22], we consider the nonlinear dependency of the longitudinal and transverse components of the dispersion on the velocity: tensor $S(v)$ is such that

$$
S(v)=S_{m} \operatorname{Id}+S_{p}(v), \quad S_{p}(v)=|v|\left(\frac{\alpha_{L}}{|v|^{2}} v \otimes v+\alpha_{T}\left(\operatorname{Id}-\frac{1}{|v|^{2}} v \otimes v\right)\right)
$$

where $S_{m}, \alpha_{L}$ and $\alpha_{T}$ are respectively the diffusion coefficient, the longitudinal and transverse dispersion factors. Here $u \otimes v$ denotes the tensor product, $(u \otimes v)_{i j}=u_{i} v_{j}$, while $u \cdot v$ denotes the scalar product, $u \cdot v=\sum_{i=1}^{N} u_{i} v_{i}$ and $|u|^{2}=u \cdot u$. The idendity matrix is denoted by Id. The eventual adsorption of the pollutant by the soil is assumed to be a linear and instantaneous reaction, following the arguments in de Marsily [19] (page 251). The corresponding retardation factor is $R$. The other reactions are resumed in the term $\tilde{r}(c)$. Classical isotherms (see e.g. Williams [25]) are described by linear functions, in the form $\tilde{r}(c)=k c$, or by Freundlich functions, $\tilde{r}(c)=k c^{k^{\prime}}$, or by Langmuir functions, $\tilde{r}(c)=k c /\left(1+k^{\prime} c\right),\left(k, k^{\prime}\right) \in \mathbb{R}_{+}^{2}$.

The model is scaled with regard to the characteristic pollutant concentrations by denoting:

$$
c=c_{r} \hat{c}
$$

where $c_{r}$ is the reference concentration. The characteristic concentration $c_{r}$ is supposed to be very small in a large part of the present paper. This assumption is frequently realistic (e.g. Commissariat général au développement durable, [27]). From the mathematical viewpoint, such a scaling justifies the perturbation analysis of the optimal control problem, depending on a small parameter. Let also $v=v_{r} \hat{v}, \phi=\phi_{r} \hat{\phi}, p=p_{r} \hat{p}$ and $g=g_{r} \hat{g}$, $S_{m}=S_{m, r} \hat{S}_{m}, S_{p}=S_{p, r} \hat{S}_{p}$. We introduce reference characteristic reaction parameters $k_{r} \in \mathbb{R}^{a}$, a $\in \mathbb{N}^{\text {, in the }}$ reaction function $\tilde{r}$ and thus get a scaled isotherm denoted by $r, r:(k, x) \in \mathbb{R}^{a} \times \mathbb{R} \mapsto r(k, x) \in \mathbb{R}$. For instance, the Freundlich and the Langmuir isotherms presented above depend on two characteristic parameters and thus correspond to a setting with $a=2$. The length scale is denoted by $x_{r}$, such that $\hat{x}=x / x_{r}$, the time scale is $t_{r}$, such that $\hat{t}=t / t_{r}$. The dimensionless form of (1)-(2) then reads

$$
\begin{gathered}
R \psi c_{r} \partial_{\hat{t}} \hat{c}+\frac{v_{r} t_{r}}{x_{r}} c_{r} \hat{v} \cdot \nabla_{\hat{x}} \hat{c}-\frac{t_{r}}{x_{r}^{2}} c_{r} \operatorname{div}_{\hat{x}}\left(\psi\left(S_{m, r} \hat{S}_{m} I d+v_{r} S_{p, r} \hat{S}_{p}(\hat{v})\right) \nabla_{\hat{x}} \hat{c}\right) \\
=-t_{r} r\left(k_{r}, c_{r} \hat{c}\right)+t_{r} p_{r} \hat{p}\left(1-c_{r} \hat{c}\right)-g_{r} t_{r} c_{r} \hat{g} \hat{c}, \\
\frac{v_{r}}{x_{r}} \operatorname{div}_{\hat{x}}(\hat{v})=p_{r} \hat{p}+g_{r} \hat{g}, \quad \hat{v}=-\frac{\phi_{r}}{v_{r} x_{r}} \kappa \nabla_{\hat{x}} \hat{\phi} .
\end{gathered}
$$


We now have to use the realistic scalings for the application we have in mind. As already mentioned, our aim is to focus on settings where the reference concentration $c_{r}$ is very small. We thus set

$$
c_{r}=\epsilon, \epsilon \ll 1
$$

We also assume that the pollutant flux is of the same order and, of course, that it is not the main process ruling the displacement in the reservoir so that the Darcy velocity remains of order one:

$$
t_{r} p_{r}=\mathcal{O}(\epsilon), \quad \frac{g_{r}}{x_{r} v_{r}}=\mathcal{O}(1), v_{r}=\mathcal{O}\left(\frac{\phi_{r}}{x_{r}}\right) .
$$

We now choose the characteristic time scale. We bear in mind that we aim to derive a model for the optimal control of polluted underground water. We thus have to select a large time scale, in the sense that any pollutant plume infiltrating the reservoir has to be taken into account until it reaches out beyond the captation wells. We thus choose the diffusion time scaling:

$$
t_{r}=\frac{x_{r}^{2}}{S_{m, r}}
$$

It is well known (e.g. [3]) that the displacement of water in a porous medium is a convection dominated process and, moreover, that dispersive effects are more important than the purely diffusive ones. With the latter diffusive scaling, we thus can consider that $v_{r} t_{r} / x_{r} \geq \mathcal{O}(1)$ and $t_{r} v_{r} S_{p, r} / x_{r}^{2}=\mathcal{O}(1)$. Notice that it means in particular that the Peclet number, $\mathrm{Pe}=x_{r} v_{r} / S_{m, r}$, is at least of order one, which is the realistic setting for this type of flow. With the latter scales, we get the following system:

$$
\begin{aligned}
& R \psi \partial_{\hat{t}}(\epsilon \hat{c})+\epsilon \hat{v} \cdot \nabla_{\hat{x}} \hat{c}-\epsilon \operatorname{div}_{\hat{x}}\left(\psi S(\hat{v}) \nabla_{\hat{x}} \hat{c}\right)=-r(\epsilon, \epsilon \hat{c})-\epsilon g \hat{c}+\epsilon \hat{p}(1-\epsilon \hat{c}), \\
& \operatorname{div}_{\hat{x}}(\hat{v})=\epsilon \hat{p}+g, \quad \hat{v}=-\kappa \nabla_{\hat{x}} \hat{\phi} .
\end{aligned}
$$

Term $r(\epsilon, \epsilon \hat{c})$ is the scaled isotherm taking into account the eventual dependance of the Damköhler number on $\epsilon$ (see e.g. Choquet and Mikelić [8] and Remark 2.4 below for explicit examples). For emphasizing the dependance of the solutions both on $\epsilon$ and on the pollutant load $p$, we denote

$$
\hat{c}=c_{\epsilon, p}, \hat{\phi}=\phi_{\epsilon, p}, \hat{v}_{p}=v_{\epsilon, p} .
$$

Let also $r_{\epsilon}$ be defined by

$$
r_{\epsilon}(c):=r(\epsilon, \epsilon c) / \epsilon .
$$

After simplification by $\epsilon$, the scaled state system finally reads (without hats for lighter notations)

$$
\begin{aligned}
& R \psi \partial_{t} c_{\epsilon, p}+v_{\epsilon, p} \cdot \nabla c_{\epsilon, p}-\operatorname{div}\left(\psi S\left(v_{\epsilon, p}\right) \nabla c_{\epsilon, p}\right)=-r_{\epsilon}\left(c_{\epsilon, p}\right)-g c_{\epsilon, p}+p\left(1-\epsilon c_{\epsilon, p}\right), \\
& \operatorname{div}\left(v_{\epsilon, p}\right)=\epsilon p+g, \quad v_{\epsilon, p}=-\kappa \nabla \phi_{\epsilon, p} .
\end{aligned}
$$

Let us now describe the optimization part of our problem. We consider a standard central planner objective, which takes into account the benefits of the polluting production (once again, the reader may bear in mind the example of the fertilizers use) on the one hand and cleaning costs on the other hand. We only address the critical case where these costs are of order one even for low concentration pollutants. The instantaneous benefits is modeled by a function $f$ depending on time, space and on the pollutant load. Cleaning costs are described by an operator $D$ depending on the position of the production wells and on the pollutant concentration $c_{\epsilon, p}$ at this position. The general form of the cleaning cost operator encompasses several properties highlighted by empirical studies. First, it has been point out by Clark and Dorsey [9] that treatment costs are site specific. As they mention no two treatment plants are alike. Costs depend on the water quality, on the cleaning technology (chemical treatments, such as disinfectants, coagulant, PH adjusters, Ultraviolet treatments, ...). Empirical studies have been launched to compute these costs. According to Roger [21], the water quality determines the 
quantity of chemicals needed to clean the water. Based on a study realized by Dearmont et al. [10], Roger explicitly obtains the cost function as a function of water turbidity, $\mathrm{pH}$ and ground-water contamination. The objective thus reads

$$
J(p)=\int_{0}^{T}\left(\int_{\Omega}\left(f(t, x, p)-D\left(x, c_{\epsilon, p}\right)\right) d x\right) e^{-\rho t} d t
$$

where $\rho \in] 0,1[$ is the social discount rate, $[0, T]$ and $\Omega$ are respectively the time and space domain of interest and $c_{\epsilon, p}$ satisfies $(4)-(5)$ completed by initial and boundary conditions.

To sum up, we state the following definition.

Definition 1.1 (Problem $\left.\mathcal{P}_{\epsilon}\right)$. Let $\epsilon>0$. Let $E_{\epsilon}$ be the set of admissible controls. Let $J$ be defined by $(6)$. Find $p_{\epsilon}^{*} \in E_{\epsilon}$ such that

$$
p_{\epsilon}^{*}=\arg \max _{p \in E_{\epsilon}} J(p)
$$

Remark 1.2. In the present paper, we assume that there is only one component of interest, the one of concentration $c$. This simple setting has been chosen for a clearer presentation of our methodology, despite a more realistic framework would of course involve several pollutants. If other soluble species are taken into account, say $n$ components, the state problem (1)-(2) takes the form

$$
\begin{aligned}
& R_{i} \psi \partial_{t} c_{i}+v \cdot \nabla c_{i}-\operatorname{div}\left(\psi S(v) \nabla c_{i}\right)=-\tilde{r}_{i}(\mathbf{c})-c_{i} \sum_{j=1}^{n} \tilde{r}_{j}(\mathbf{c}) \\
& \quad+p_{i}\left(1-c_{i}\right)-c_{i} \sum_{j=1, j \neq i}^{n} p_{j}+g_{i}-c_{i} \sum_{j=1}^{n} g_{j}, \quad i=1, \ldots n-1, \\
& \operatorname{div}(v)=\sum_{j=1}^{n}\left(p_{j}+g_{j}\right)-\sum_{j=1}^{n} \tilde{r}_{j}(\mathbf{c}), \quad v=-\kappa \nabla \phi
\end{aligned}
$$

where we set $\mathbf{c}=\left(c_{1}, \ldots, c_{n}\right)$ and $c_{n}=1-\sum_{i=1}^{n-1} c_{i}$. On the one hand, comparing the structure of this model with the one of (1)-(2), one easily guess that the mathematical analysis providing an existence result is very similar for both systems. But on the other hand, an hypothetical uniqueness result is very dependent of the form of the reaction terms $\tilde{r}_{j}$ coupling nonlinearly all the equations. It thus requires a case-by-case analysis according to each chemical kinetics. At the end of the present paper, the reader will be probably convinced that the assumptions on the reaction functions $\tilde{r}_{j}$ are also crucial for stating a uniqueness result for each optimal control problem in the form $J_{i}\left(p_{i}\right)=\int_{0}^{T}\left(\int_{\Omega}\left(f_{i}\left(t, x, p_{i}\right)-D_{i}\left(x, c_{i}\right)\right) d x\right) e^{-\rho t} d t$ that may be associated to the state system. The uniqueness of the optimal control for a global functional in the form $J\left(p_{1}, \cdots, p_{n}\right)=\sum_{i=1}^{n} J_{i}\left(p_{i}\right)$ is in general out of reach.

\section{Mathematical Assumptions and main Results}

Space is limited to a bounded domain $\Omega \subset \mathbb{R}^{N}, N \leq 3$, representing an area containing both the pollution source and groundwater collection wells. We assume that the boundary $\partial \Omega$ of $\Omega$ is such that $\partial \Omega \in \mathcal{C}^{2}$. Time horizon is denoted by $T$, with a small abuse of notation allowing

$$
0<T \leq \infty
$$

Let $\Omega_{T}=\Omega \times(0, T)$. 
We begin with a mathematical setting ensuring, at least, an existence result of weak solutions for the state system (4)-(5). It is completed by the following initial and boundary conditions. Here we choose Neumann conditions for $c$ (no-flux) and Dirichlet conditions for $\phi$, namely

$$
\begin{aligned}
& S\left(v_{\epsilon, p}\right) \nabla c_{\epsilon, p} \cdot n=0 \text { on } \partial \Omega \times(0, T), \\
& \phi_{\epsilon, p}=\phi_{1} \text { on } \partial \Omega \times(0, T),
\end{aligned}
$$

where $n$ is the unit exterior normal to $\partial \Omega$. As mentioned in [1], other boundary conditions could be treated with slight modifications in our computations. The initial condition is

$$
c_{\mid t=0}=c_{0} \text { in } \Omega \text {. }
$$

We assume

$$
S_{m}>0, \quad 0 \leq \alpha_{T} \leq \alpha_{L}, \quad R>0
$$

and that there exist real numbers $\psi_{-}, \psi_{+}$, and $0<\kappa_{-} \leq \kappa_{+}$, such that

$$
\begin{aligned}
& 0<\psi_{-} \leq \psi(x) \leq \psi_{+} \text {a.e. } x \in \Omega, \\
& \kappa \xi \cdot \xi \geq \kappa_{-}|\xi|^{2} \text { and }|\kappa \xi| \leq \kappa_{+}|\xi| \text { in } \Omega, \forall \xi \in \mathbb{R}^{N} .
\end{aligned}
$$

Function $g$ is nonnegative and

$$
(t, x) \mapsto g(t, x, p(t, x)) \in L^{\infty}\left(\Omega_{T}\right) \text { for any } p \in L^{\infty}\left(\Omega_{T}\right)
$$

We assume $\phi_{1} \in L^{\infty}\left(0, T ; W^{2-1 / q, q}(\partial \Omega)\right)$, so that, in view of the smoothness of $\partial \Omega$, there exists a lifting of $\phi_{1}$, still denoted the same for convenience, such that

$$
\phi_{1} \in L^{\infty}\left(0, T ; W^{2, q}(\Omega)\right)
$$

where the value of $q$ depends on another assumption, for $\kappa$, namely:

$$
\begin{array}{cl}
q>N & \text { if } \kappa \in\left(\mathcal{C}^{1}(\bar{\Omega})\right)^{N \times N}, \\
q>N / 2 & \text { if } \kappa=\tilde{\kappa} \operatorname{Id}, \tilde{\kappa} \in \mathcal{C}^{1}(\bar{\Omega} ; \mathbb{R}) .
\end{array}
$$

The existence of the lifting of the boundary condition $\phi_{1}$ with the appropriate regularity is ensured by the surjectivity of the trace operator from $W^{2, q}(\Omega)$ onto $W^{2-1 / q, q}(\partial \Omega)$ (see [18] for the case $q=2$ and the references therein for $q \neq 2)$. Initial data $c_{0} \in L^{\infty}(\Omega)$ is in the admissible range for a concentration

$$
0 \leq c_{0}(x) \leq 1 \text { a.e. } x \in \Omega
$$

For any $\epsilon>0$, the reaction term $x \mapsto r(\epsilon, x)$ is defined in the same range of concentrations, the interval $[0,1]$. It is assumed Lipschitz in $[0,1]$ and such that

$$
r(\epsilon, 0)=0, r(\epsilon, 1)+g \geq 0 \text {, for any } 0<\epsilon<\epsilon_{0} \text { where } \epsilon_{0}>0 .
$$

One checks easily that classical isotherms satisfy this technical assumption (see Bear [3]), which is set for ensuring a physical maximum principle for the concentrations.

We are now in a position to recall the following existence result of global weak solutions for the state system. We refer to [1], Proposition 3.1 and Lemma 3.1, for its proof. 
Definition 2.1. A couple $\left(c_{\epsilon, p}, \phi_{\epsilon, p}\right)$, with $c_{\epsilon, p} \in L^{2}\left(0, T ; H^{1}(\Omega)\right)$ and $\phi_{\epsilon, p} \in L^{\infty}\left(0, T ; H^{2}(\Omega)\right)$, is a weak solution of (4)-(5), (7)-(9), if for any test function $\varphi \in H^{1}\left(0, T ; H^{1}(\Omega)\right)$ with $\varphi_{\mid t=T}=0$,

$$
\begin{aligned}
& -\int_{\Omega_{T}} R \psi c_{\epsilon, p} \partial_{t} \varphi d x d t-\int_{\Omega} R \psi c_{0} \varphi_{\mid t=0} d x+\int_{\Omega_{T}}\left(v_{\epsilon, p} \cdot \nabla c_{\epsilon, p}\right) \varphi d x d t \\
& \quad+\int_{\Omega_{T}} \psi S\left(v_{\epsilon, p}\right) \nabla c_{\epsilon, p} \cdot \nabla \varphi d x d t=\int_{\Omega_{T}}\left(-r_{\epsilon}\left(c_{\epsilon, p}\right)+p_{\epsilon}\left(1-\epsilon c_{\epsilon, p}\right)-g c_{\epsilon, p}\right) \varphi d x d t \\
& \int_{\Omega_{T}} \operatorname{div} v_{\epsilon, p} \varphi d x d t=\int_{\Omega_{T}}(\epsilon p+g) \varphi d x d t
\end{aligned}
$$

and, for all $\varphi^{\prime} \in L^{1}\left(0, T ; H_{0}^{1}(\Omega)\right)$,

$$
\int_{\Omega_{T}} v_{\epsilon, p} \cdot \nabla \varphi^{\prime} d x d t=\int_{\Omega_{T}}-\kappa \nabla \phi_{\epsilon, p} \cdot \nabla \varphi^{\prime} d x d t
$$

with moreover $\phi_{\epsilon, p}=\phi_{1}$ on $\partial \Omega \times(0, T)$ in the trace sense.

Proposition 2.2. For any given $p \in L^{\infty}\left(\Omega_{T}\right)$ and any $0 \leq \epsilon<\epsilon_{0}$, there exists a unique global weak solution $v_{\epsilon, p} \in\left(L^{\infty}\left(\Omega_{T}\right)\right)^{N}$ of (5), (8) and a weak global solution $c_{\epsilon, p} \in \mathcal{C}\left(0, T ; L^{2}(\Omega)\right) \cap L^{2}\left(0, T ; H^{1}(\Omega)\right)$ of (4), (7), (9). If $\epsilon>0$, it satisfies moreover $0 \leq c_{\epsilon, p}(t, x) \leq 1 / \epsilon$ almost everywhere in $\Omega_{T}$.

Let us now describe the assumptions linked with the optimization part of the problem. A natural admissible set of control should be

$$
E_{0}=\left\{p \in L^{2}\left(\Omega_{T}\right) ; 0 \leq p(t, x) \leq \bar{p} \text { a.e. in } \Omega_{T}\right\}
$$

where $\bar{p}$ is some arbitrary real number, but for technical reasons we are going to look for an optimal control belonging to the subset $E_{\epsilon} \subset E_{0}$ defined by

$$
E_{\epsilon}=\left\{p \in L^{2}\left(\Omega_{T}\right) \cap W^{1,1}(0, T ; X) ; 0 \leq p(x, t) \leq \bar{p} \text { a.e. in } \Omega_{T},\left\|\epsilon \alpha_{\epsilon} \partial_{t} p\right\|_{L^{1}(0, T ; X)} \leq C_{p}\right\}
$$

where $C_{p} \geq 0$ is a given real number, $X$ is a Banach functional space such that the embedding $L^{2}(\Omega) \subset X$ is continuous, and $\alpha_{\epsilon}$ is defined by

$$
\alpha_{\epsilon}=\left\{\begin{array}{l}
0 \text { if } \epsilon p+g \text { does not depend on } p \\
1 \text { otherwise. }
\end{array}\right.
$$

It means that $\alpha_{\epsilon}$ depends on the influence of the pollutant concentration on the incompressibility equation. In the $\epsilon$-dependent problem, the restriction $E_{\epsilon}$ of the set of controls $E_{0}$ is introduced for ensuring a compactness result despite its coupling with the unknown control $p_{\epsilon}$ in equation (13). The restriction disappears as $\epsilon \rightarrow 0$. Notice the compatibility in the notations since actually $E_{0}=E_{\epsilon}$ for $\epsilon=0$. The other technical assumptions are the following. Function $p \in[0, \bar{p}] \mapsto f(t, x, p)$ is strictly concave, upper semi-continuous and bounded almost everywhere in $\Omega_{T}$. For almost every $x \in \Omega$, the operator $c \in L^{2}\left(\Omega_{T}\right) \mapsto D(x, c) \in\left(L^{2}\left(\Omega_{T}\right)\right)^{\prime}=L^{2}\left(\Omega_{T}\right)$ is convexe, monotone, hemicontinuous and bounded in the sense that there exists $h \in L^{1}\left(\Omega_{T}\right)$ such that $\|D(x, c)\|_{L^{2}\left(\Omega_{T}\right)} \leq h(t, x)$ almost everywhere in $\Omega_{T}$, for any $c \in L^{2}\left(\Omega_{T}\right)$.

Bear in mind that Problem $\mathcal{P}_{\epsilon}$ defined in (1.1) consists in finding $\left(p_{\epsilon}^{*}, c_{\epsilon}^{*}, \phi_{\epsilon}^{*}\right)$ such that

$$
J\left(p_{\epsilon}^{*}\right)=\max _{p_{\epsilon} \in E_{\epsilon}} J\left(p_{\epsilon}\right)
$$

and $\left(c_{\epsilon}^{*}=c_{\epsilon, p_{\epsilon}^{*}}, \phi_{\epsilon}^{*}=\phi_{\epsilon, p_{\epsilon}^{*}}\right)$ is a weak solution of (4)-(5), (7)-(9), associated to $p_{\epsilon}^{*}$ in the sense of Definition 2.1. The first main result of the paper is the following existence result for $\mathcal{P}_{\epsilon}$. 
Theorem 2.3. Let $0<\epsilon<\epsilon_{0}$. There exists a global solution $\left(p_{\epsilon}^{*}, c_{\epsilon}^{*}, \phi_{\epsilon}^{*}\right)$ to the optimal control problem $\mathcal{P}_{\epsilon}$ in the sense of Definition 1.1, such that $c_{\epsilon}^{*} \in \mathcal{C}\left(0, T ; L^{2}(\Omega)\right) \cap L^{2}\left(0, T ; H^{1}(\Omega)\right), v_{\epsilon}^{*} \in\left(L^{\infty}\left(\Omega_{T}\right)\right)^{N}$ and $0 \leq c_{\epsilon}^{*}(x, t) \leq 1 / \epsilon$ almost everywhere in $\Omega_{T}$.

The second aim of the article consists in the analysis of the behavior of the optimal control problem $\mathcal{P}_{\epsilon}$ when the characteristic pollutant concentration is small. More precisely, we derive rigorously the corresponding effective model by letting $\epsilon$ tend to zero. An important point is to state assumptions for the reaction term $r_{\epsilon}$ which are sufficient to pass to the limit, but nevertheless as generic as possible. We simply assume that:

the sequence $r_{\epsilon}$ converges pointwise to some function $r_{0}$ when $\epsilon$ tends to 0 ; the function $r_{0}$ is assumed to be concave, derivable and has a bounded derivative on $\mathbb{R}_{+}$.

Remark 2.4. In the simplest setting, the isotherm of the scaled model only depends on the second variable, that is $r(\epsilon, \epsilon x)=r(\epsilon x), x \in[0,1]$. By definition of $r_{\epsilon}$, the existence of $r_{0}$ is equivalent to the existence of $r^{\prime}(0)$, which is not an additional assumption. Moreover, for $\epsilon c_{\epsilon}$ in the nearby of 0, using a Taylor expansion, we can write $r\left(\epsilon c_{\epsilon}\right)=r(0)+\epsilon c_{\epsilon} r^{\prime}(0)+\epsilon c_{\epsilon} \eta\left(\epsilon c_{\epsilon}\right)$ with $\lim _{\epsilon c_{\epsilon} \rightarrow 0} \eta\left(\epsilon c_{\epsilon}\right)=0$. In the present paper we have set $r(0)=0$. Then, if $c_{\epsilon} \rightarrow c, r_{\epsilon}\left(c_{\epsilon}\right)=r\left(\epsilon c_{\epsilon}\right) / \epsilon$ tends to $r^{\prime}(0) c:=r_{0}(c)$. We thus get at the limit a linear isotherm. But taking into account the potential dependance of $r$ on $\epsilon$, i.e. $r(\epsilon, \epsilon x)$, we have to consider the possibility of various isotherms at the limit. Let us give two simple examples. First, in (1), assume that $\tilde{r}$ corresponds to a Freundlich isotherm, $\tilde{r}(x)=k x^{1 / m}, k \in \mathbb{R}_{+}, m>1$. For the dimensionless equation (4), we define a characteristic reaction parameter $k_{r}$ so that $r_{\epsilon}(c)=t_{r} k_{r} k \epsilon^{1 / m-1} c^{1 / m}$. The sequence converges pointwise to the Freundlich function, $r_{0}=\tilde{r}$, if $k_{r}$ is small, namely such that $t_{r} k_{r}=\mathcal{O}\left(\epsilon^{1-1 / m}\right)$. The Langmuir isotherm function $r_{0}(c)=\tilde{r}(c)=k_{1} c /\left(1+k_{2} c\right),\left(k_{1}, k_{2}\right) \in \mathbb{R}_{+}^{2}$, may also be considered by setting $t_{r} k_{1, r}=\mathcal{O}(1)$ and $k_{2, r}=\mathcal{O}\left(\epsilon^{-1}\right)$. Notice that all these scalings are physically admissible (see [13]).

We claim that the effective problem corresponding to the limit $\epsilon \rightarrow 0$ in $\mathcal{P}_{\epsilon}$ is the following.

Definition 2.5 (Problem $\left.\mathcal{P}_{0}\right)$. Find $p^{*} \in E_{0}$ such that

$$
p^{*}=\arg \max _{p \in E_{0}} J(p)
$$

where $J$ remains defined by $(6)$, that is $J(p)=\int_{0}^{T}\left(\int_{\Omega}\left(f(t, x, p)-D\left(x, c_{p}\right)\right) d x\right) e^{-\rho t} d t$, with $c_{p}$ a weak solution of the following effective state problem:

$$
\begin{aligned}
& R \psi \partial_{t} c_{p}-\operatorname{div}\left(\psi S(v) \nabla c_{p}\right)+v \cdot \nabla c_{p}=-r_{0}\left(c_{p}\right)+p-g c_{p} \text { in } \Omega \times(0, T), \\
& \operatorname{div}(v)=g, v=-\kappa \nabla \phi \text { in } \Omega \times(0, T), \\
& S(v) \nabla c_{p} \cdot n=0 \text { on } \partial \Omega \times(0, T), c_{p \mid t=0}=c_{0} \text { in } \Omega, \\
& \phi=\phi_{1} \text { on } \partial \Omega \times(0, T) .
\end{aligned}
$$

On the one hand, when comparing $\mathcal{P}_{\epsilon}$ and $\mathcal{P}_{0}$, one notices straightforward that $E_{\epsilon}$ tends to $E_{0}$ as $\epsilon \rightarrow 0$. On the other hand, choosing $E_{0}$ in Problem $\mathcal{P}_{0}$ is fully consistent with the (announced) limit state problem since the incompressibility equation (19) does not depend on $p$, thus leading to $\alpha_{0}=0$ in definition (16). Furthermore, we state and prove below the following results.

Theorem 2.6. (i) There exists a unique global optimal solution $p^{*} \in E_{0}$ to Problem $\mathcal{P}_{0}$. It is associated to $\left(c^{*}:=c_{p^{*}}, \phi\right)$ defined by (17)-(20). Moreover $c^{*}(t, x) \geq 0$ almost everywhere in $\Omega_{T}$ and $c^{*} \in \mathcal{C}\left(0, T ; L^{2}(\Omega)\right) \cap$ $L^{2}\left(0, T ; H^{1}(\Omega)\right), v^{*} \in\left(L^{\infty}\left(\Omega_{T}\right)\right)^{N}$.

(ii) As $\epsilon \rightarrow 0$, any sequence of optimal solutions $\left(p_{\epsilon}^{*}, c_{\epsilon}^{*}, \phi_{\epsilon}^{*}\right)$ of Problem $\mathcal{P}_{\epsilon}$ converges to the optimal solution $\left(p^{*}, c^{*}, \phi\right)$ of $\mathcal{P}_{0}$ :

$$
\begin{aligned}
& c_{\epsilon}^{*} \rightarrow c^{*} \text { weakly in } L^{2}\left(0, T ; H^{1}(\Omega)\right), \quad c_{\epsilon}^{*} \rightarrow c^{*} \text { in } L^{2}\left(\Omega_{T}\right) \text { and a.e. in } \Omega_{T}, \\
& v_{\epsilon}^{*} \rightarrow v \text { in }\left(L^{2}\left(\Omega_{T}\right)\right)^{N}, \quad \phi_{\epsilon}^{*} \rightarrow \phi \text { in } L^{s}\left(0, T ; W^{1, s}(\Omega)\right) \text { for any } s \geq 1 .
\end{aligned}
$$


Theorems 2.3 and 2.6 are proved in the three following sections. We begin in Section 3 by a preliminary stability analysis of the state system. Indeed, several steps of the proofs involve the study of the limit behavior of perturbations of the optimal control problem, thus in particular of the state system. The stability properties are first used in Section 4 for stating an existence result for an optimal control problem corresponding to the generic form of Problem $\mathcal{P}_{\epsilon}$ and Problem $\mathcal{P}_{0}$. The proof is based on the sequential definition of the supremum. It turns out that the generic statement contains as particular cases the existence result in Theorem 2.3 and the local-in-time version of the one in Theorem 2.6 (i). We then prove the uniqueness of the solution of Problem $\mathcal{P}_{0}$ by contradiction. The global existence result for $\mathcal{P}_{0}$ and Theorem 2.6 (ii) are obtained at once in Section 5 , by studying the limit behavior of the global solution of Problem $\mathcal{P}_{\epsilon}$.

\section{Preliminary: Stability Results For the STATE PROBlem}

In the present section we study the stability properties of any weak solution of the state problem, (4)-(5), (7)-(9), regarding the variations of $\epsilon$ and $p$. We begin by stating some uniform estimates. Here and below, letter $C$ denotes a generic constant.

Proposition 3.1. Let $0<\epsilon<\epsilon_{0}$. Let $p \in E_{\epsilon}$. Any weak solution $\left(c_{\epsilon, p}, \phi_{\epsilon, p}\right)$ of (4)-(5) completed by (7)-(9), satifies the following estimates, uniform with regard to $p$ and $\epsilon$ :

$$
\begin{aligned}
& \left\|\phi_{\epsilon, p}\right\|_{L^{\infty}\left(0, T ; W^{1, \infty}(\Omega)\right)} \leq C, \\
& \left\|c_{\epsilon, p}\right\|_{L^{\infty}\left(0, T ; L^{2}(\Omega)\right) \cap L^{2}\left(0, T ; H^{1}(\Omega)\right)} \leq C, \\
& \left\|\psi \partial_{t} c_{\epsilon, p}\right\|_{L^{2}\left(0, T ;\left(H^{1}(\Omega)\right)^{\prime}\right)} \leq C .
\end{aligned}
$$

Proof. We begin by the results on the hydraulic head $\phi_{\epsilon, p}$. Multiplying (5) by $\phi_{\epsilon, p}-\phi_{1}$ and integrating by parts over $\Omega$, we get

$$
\int_{\Omega} \kappa \nabla \phi_{\epsilon, p} \cdot \nabla \phi_{\epsilon, p} d x=\int_{\Omega} \kappa \nabla \phi_{\epsilon, p} \cdot \nabla \phi_{1} d x+\int_{\Omega}(\epsilon p+g)\left(\phi_{\epsilon, p}-\phi_{1}\right) d x
$$

Using the assumptions on $\kappa, \phi_{1}, g$ and the Cauchy-Schwarz and Young inequalities, bearing in mind that $p \in E_{\epsilon}$ satisfies $0 \leq p \leq \bar{p}$ a.e. in $\Omega_{T}$, we infer from the latter relation that

$$
\kappa_{-} \int_{\Omega}\left|\nabla \phi_{\epsilon, p}\right|^{2} d x \leq \eta \int_{\Omega}\left|\nabla \phi_{\epsilon, p}\right|^{2} d x+\eta \int_{\Omega}\left|\phi_{\epsilon, p}-\phi_{1}\right|^{2} d x+\frac{C}{\eta}
$$

for any $\eta>0$. Next, using the Poincaré inequality we write $\eta\left\|\phi_{\epsilon, p}-\phi_{1}\right\|_{L^{2}(\Omega)}^{2} \leq \eta C\left\|\nabla \phi_{\epsilon, p}-\nabla \phi_{1}\right\|_{\left(L^{2}(\Omega)\right)^{N}}^{2} \leq$ $\eta C\left\|\nabla \phi_{\epsilon, p}\right\|_{\left(L^{2}(\Omega)\right)^{N}}^{2}+C$. Choosing $\eta$ sufficiently small, we obtain the following uniform estimate:

$$
\left\|\phi_{\epsilon, p}\right\|_{L^{\infty}\left(0, T ; H^{1}(\Omega)\right)} \leq C .
$$

Using the regularity assumptions set on $\kappa$ and $\phi_{1}$, let us improve the result. Setting $f=\epsilon p+g+\operatorname{div}\left(\kappa \nabla \phi_{1}\right)$, $u_{\epsilon, p}=\phi_{\epsilon, p}-\phi_{1}$ solves the homogenous Dirichet problem

$$
\operatorname{div}\left(-\kappa \nabla u_{\epsilon, p}\right)=f \text { in } \Omega_{T}, u_{\epsilon, p}=0 \text { on } \partial \Omega \times(0, T) .
$$

Since $\phi_{1} \in L^{\infty}\left(0, T ; W^{2, q}(\bar{\Omega})\right)$, then $f \in L^{\infty}\left(0, T ; L^{q}(\Omega)\right)$. According to the Nirenberg's theorem (see e.g. [16]), it follows that $u_{\epsilon, p} \in L^{\infty}\left(0, T ; W_{0}^{2, q}(\Omega)\right)$. This point allows to multiply (25), written in the form $-\kappa \Delta u_{\epsilon, p}-$ $\nabla \kappa \cdot \nabla u_{\epsilon, p}=f$, by $\left|\Delta u_{\epsilon, p}\right|^{s-2} \Delta u_{\epsilon, p}, s \leq q$, and to integrate by parts in $\Omega$. We obtain

$$
\kappa_{-} \int_{\Omega}\left|\Delta u_{\epsilon, p}\right|^{s} d x \leq\left.\left|\int_{\Omega}\right| \Delta u_{\epsilon, p}\right|^{s-2} \Delta u_{\epsilon, p}\left(\nabla \kappa \cdot \nabla u_{\epsilon, p}\right) d x|+| \int_{\Omega}\left|\Delta u_{\epsilon, p}\right|^{s-2} \Delta u_{\epsilon, p} f d x \mid .
$$


We compute

$$
\begin{aligned}
& \left.\left.\left|\int_{\Omega}\right| \Delta u_{\epsilon, p}\right|^{s-2} \Delta u_{\epsilon, p}\left(\nabla \kappa \cdot \nabla u_{\epsilon, p}+f\right) d x\left|\leq \int_{\Omega}\right| \Delta u_{\epsilon, p}\right|^{s-1}\left(|| \nabla \kappa \|_{L^{\infty}(\Omega)}\left|\nabla u_{\epsilon, p}\right|+|f|\right) d x \\
& \quad \leq C\left(\int_{\Omega}\left|\Delta u_{\epsilon, p}\right|^{s} d x\right)^{(s-1) / s}\left(\int_{\Omega}\left|\nabla u_{\epsilon, p}+f\right|^{s} d x\right)^{1 / s} \leq \eta \int_{\Omega}\left|\Delta u_{\epsilon, p}\right|^{s} d x+\frac{C}{\eta} \int_{\Omega}\left|\nabla u_{\epsilon, p}+f\right|^{s} d x
\end{aligned}
$$

for any $\eta>0$. The latter relation in (26) gives, by choosing $\eta$ small enough: for any $s \leq q$,

$$
\int_{\Omega}\left|\Delta u_{\epsilon, p}\right|^{s} d x \leq C \int_{\Omega}\left|\nabla u_{\epsilon, p}+f\right|^{s} d x
$$

Now, our computations somehow differs according to whether we use the assumption $q>N$ or the assumption $q>N / 2$ with an isotropic diffusion, that is $\kappa=\tilde{\kappa}$ Id with $\tilde{\kappa} \in \mathcal{C}^{1}(\bar{\Omega})$.

Assume first $q>N$. Because of (24), we first infer from the relation (27) for $s=2$ that $\left\|\Delta u_{\epsilon, p}\right\|_{L^{\infty}\left(0, T ; L^{2}(\Omega)\right)} \leq$ $C$. It means that there exists $\tilde{f} \in L^{\infty}\left(0, T ; L^{2}(\Omega)\right),\|\tilde{f}\|_{L^{\infty}\left(0, T ; L^{2}(\Omega)\right)} \leq C$, such that $u_{\epsilon, p}$ satisfies

$$
\Delta u_{\epsilon, p}=\tilde{f} \text { in } \Omega_{T}, u_{\epsilon, p}=0 \text { on } \partial \Omega \times(0, T) .
$$

Thus $u_{\epsilon, p}$ is uniformly bounded in $L^{\infty}\left(0, T ; H_{0}^{2}(\Omega)\right)$. In particular, $\nabla u_{\epsilon, p}$ is uniformly bounded in the space $\left(L^{\infty}\left(0, T ; H_{0}^{1}(\Omega)\right)\right)^{N}$, and, due to classical Sobolev's embeddings, in $\left(L^{\infty}\left(0, T ; L^{4}(\Omega)\right)\right)^{N}$. Choosing now $s=$ $\min (q, 4)$ in $(27)$, we get

$$
\left\|\Delta u_{\epsilon, p}\right\|_{L^{\infty}\left(0, T ; L^{s}(\Omega)\right)} \leq C .
$$

The same uniform estimate holds true for $\phi_{\epsilon, p}=u_{\epsilon, p}+\phi_{1}$ because of the smoothness of $\phi_{1}$. Since $s>N$, the latter result is sufficient for proving (21), once again thanks to Nirenberg's theorem and to classical Sobolev's embeddings.

Assume now $q>N / 2$ and $\kappa=\kappa^{*} I d, \kappa^{*} \in C^{1}(\bar{\Omega})$. Because of $(24)$, choosing $s=\min (q, 2)$ in (27), we first obtain a uniform bound for $\Delta u_{\epsilon, p}$ in $L^{\infty}\left(0, T ; L^{s}(\Omega)\right)$. Assume for simplicity that $\min (q, 2)=q$. It follows that $u_{\epsilon, p}$ is uniformly bounded in $L^{\infty}\left(0, T ; W_{0}^{2, q}(\Omega)\right)$. The same holds true for $\phi_{\epsilon, p}=u_{\epsilon, p}+\phi_{1}$. This bound and the specific structure of $\kappa$ let us compute that $\left\|\operatorname{curl}\left(v_{\epsilon, p}\right)\right\|_{\left(L^{\infty}\left(0, T ; W^{1, q}(\Omega)\right)\right)^{N}}=\left\|\nabla \kappa \wedge \nabla \phi_{\epsilon, p}\right\|_{\left(L^{\infty}\left(0, T ; W^{1, q}(\Omega)\right)\right)^{N}} \leq C$. In particular, due to classical Sobolev's embedding, $\left\|\operatorname{curl}\left(v_{\epsilon, p}\right)\right\|_{\left(L^{\infty}\left(0, T ; L^{q_{0}}(\Omega)\right)\right)^{N}} \leq C$ for some $q_{0}>N$ since $q>N / 2$. We also know that $\left\|\operatorname{div}\left(v_{\epsilon, p}\right)\right\|_{L^{\infty}\left(0, T ; L^{q_{0}}(\Omega)\right)}=\|\epsilon p+g\|_{L^{\infty}\left(\Omega_{T}\right)} \leq C$. Recall that $H_{q_{0}}(\operatorname{curl}, \operatorname{div}, \Omega):=$ $\left\{u \in\left(L^{q_{0}}(\Omega)\right)^{N} ; \operatorname{curl}(u) \in\left(L^{q_{0}}(\Omega)\right)^{N}, \operatorname{div}(u) \in L^{q_{0}}(\Omega)\right\}$ is a Hilbert space when endowed with the norm

$$
\left(\|u\|_{\left(L^{q_{0}}(\Omega)\right)^{N}}+\|\operatorname{curl}(u)\|_{\left(L^{q_{0}}(\Omega)\right)^{N}}+\|\operatorname{div}(u)\|_{L^{q_{0}}(\Omega)}\right)^{1 / q_{0}}
$$

and is such that $H_{q_{0}}(\operatorname{curl}, \operatorname{div}, \Omega) \subset\left(W_{l o c}^{1, q_{0}}(\Omega)\right)^{N}$. Here we thus claim that $v_{\epsilon, p}$ is uniformly bounded in the space $\left(L^{\infty}\left(0, T ; W_{l o c}^{1, q_{0}}(\Omega)\right)\right)^{N}$ where $q_{0}>N$. Then it follows from Rellich-Kondrachov theorem that $v_{\epsilon, p}$ is also uniformly bounded in $\left(L^{\infty}\left(\Omega_{T}\right)\right)^{N}$.

We now look for the uniform estimates of the concentration. Let $\tau \in] 0, T\left[\right.$. We multiply $(4)$ by $c_{\epsilon, p}(t, x) \chi_{[0, \tau]}(t)$, $\chi_{U}$ denoting the characteristic function of a set $U$, and we integrate by parts over $\Omega_{T}$. Thanks to the definition of tensor $S\left(v_{\epsilon, p}\right)$, we obtain

$$
\begin{aligned}
& \frac{R}{2} \int_{\Omega} \psi\left|c_{\epsilon, p}(\tau, x)\right|^{2} d x+\int_{0}^{\tau} \int_{\Omega} \psi_{-}\left(S_{m}+\alpha_{T}\left|v_{\epsilon, p}\right|\right)\left|\nabla c_{\epsilon, p}\right|^{2} d x d t+\int_{0}^{\tau} \int_{\Omega}\left(v_{\epsilon, p} \cdot \nabla c_{\epsilon, p}\right) c_{\epsilon, p} d x d t \\
& \quad+\int_{0}^{\tau} \int_{\Omega} r_{\epsilon}\left(c_{\epsilon, p}\right) c_{\epsilon, p} d x d t+\int_{0}^{\tau} \int_{\Omega}(g+\epsilon p) c_{\epsilon, p}^{2} d x d t-\int_{0}^{\tau} \int_{\Omega} p c_{\epsilon, p} d x d t \leq \frac{R}{2} \int_{\Omega} \psi\left|c_{0}\right|^{2} d x .
\end{aligned}
$$


With Cauchy-Schwarz and Young inequalities, we get straightforward

$$
\left|\int_{0}^{\tau} \int_{\Omega} r_{\epsilon}\left(c_{\epsilon, p}\right) c_{\epsilon, p} d x d t+\int_{0}^{\tau} \int_{\Omega}(g+\epsilon p) c_{\epsilon, p}^{2} d x d t-\int_{0}^{\tau} \int_{\Omega} p c_{\epsilon, p} d x d t\right| \leq C+C \int_{0}^{\tau} \int_{\Omega}\left|c_{\epsilon, p}\right|^{2} d x d t .
$$

The main difficulty traditionally lies in the estimate of the convective term. Here, we avoid it thanks to the work already done for getting a uniform estimate for velocity $v_{\epsilon, p}$ in $\left(L^{\infty}\left(\Omega_{T}\right)\right)^{N}$ :

$$
\begin{gathered}
\left|\int_{0}^{\tau} \int_{\Omega}\left(v_{\epsilon, p} \cdot \nabla c_{\epsilon, p}\right) c_{\epsilon, p} d x d t\right| \leq\left\|v_{\epsilon, p}\right\|_{\left(L^{\infty}\left(\Omega_{T}\right)\right)^{N}}\left\|\nabla c_{\epsilon, p}\right\|_{\left(L^{2}(\Omega \times(0, \tau))\right)^{N}}\left\|c_{\epsilon, p}\right\|_{L^{2}(\Omega \times(0, \tau))} \\
\leq \eta \int_{0}^{\tau}\left|\nabla c_{\epsilon, p}\right|^{2} d x d t+\frac{C}{\eta} \int_{0}^{\tau}\left|c_{\epsilon, p}\right|^{2} d x d t
\end{gathered}
$$

for any $\eta>0$. Insert the two latter relations in (28) and choose $\eta=\psi_{-} S_{m} / 2$ :

$$
\frac{R \psi_{-}}{2} \int_{\Omega}\left|c_{\epsilon, p}(\tau, x)\right|^{2} d x+\frac{\psi_{-}}{2} \int_{0}^{\tau} \int_{\Omega}\left(S_{m}+\alpha_{T}\left|v_{\epsilon, p}\right|\right)\left|\nabla c_{\epsilon, p}\right|^{2} d x d t \leq C+C \int_{0}^{\tau} \int_{\Omega}\left|c_{\epsilon, p}(t, x)\right|^{2} d x d t .
$$

We then get (22) using Gronwall's Lemma.

Now we check that $\psi \partial_{t} c_{\epsilon, p}$ is uniformly bounded in $L^{2}\left(0, T ;\left(H^{1}(\Omega)\right)^{\prime}\right)$. Multiply (4) by some test function $\varphi$ in $L^{2}\left(0, T ; H^{1}(\Omega)\right)$ and integrate by parts over $\Omega_{T}$. Bearing in mind the latter estimates and that $0 \leq c_{\epsilon, p}(t, x) \leq$ $1 / \epsilon$ almost everywhere in $\Omega_{T}$, we obtain straightforward

$$
\left|\left\langle\psi \partial_{t} c_{\epsilon, p}, \varphi\right\rangle_{L^{2}\left(0, T ;\left(H^{1}(\Omega)\right)^{\prime}\right) \times L^{2}\left(0, T ; H^{1}(\Omega)\right)}\right|=\left|\int_{\Omega_{T}} \psi \partial_{t} c_{\epsilon, p} \varphi d x d t\right| \leq C .
$$

Here, we denote by $\langle\cdot, \cdot\rangle_{X^{\prime} \times X}$ the duality pair defined by $\langle u, v\rangle_{X^{\prime} \times X}=\int_{\Omega_{T}} u v d x d t$ if $X$ is a space of functions defined in $\Omega_{T}, u \in X^{\prime}, v \in X$. Proposition 3.1 is proved.

Now we state and prove some compactness results. Let $\left(\epsilon_{n}\right)_{n \in \mathbb{N}^{*}}$ and $\left(p_{n}\right)_{n \in \mathbb{N}^{*}}$ be two sequences of, respectively, $\mathbb{R}_{+}^{*}$ and $E_{\epsilon_{n}}$. Denote $\phi_{n}=\phi_{\epsilon_{n}, p_{n}}, v_{n}=v_{\epsilon_{n}, p_{n}}, c_{n}=c_{\epsilon_{n}, p_{n}}$. Assume that

$$
\epsilon_{n} \rightarrow \epsilon_{0} \text { in } \mathbb{R} \text { and } p_{n} \rightarrow p_{0} \text { weakly in } L^{2}\left(\Omega_{T}\right)
$$

as $n$ tends to infinity.

Proposition 3.2. The following sequential compactness results hold true, up to subsequences not relabeled for convenience: there exists $v \in\left(L^{\infty}\left(\Omega_{T}\right)\right)^{N}$ and $c \in L^{2}\left(0, T ; H^{1}(\Omega)\right)$ such that

$$
c_{n} \rightarrow c \text { in } L^{s}\left(0, T ; L^{2}(\Omega)\right), 1 \leq s<2 \text {, and a.e. in } \Omega_{T}
$$

and

as $n$ tends to infinity.

$$
v_{n} \rightarrow v \text { in }\left(L^{2}\left(\Omega_{T}\right)\right)^{N} \text { and a.e. in } \Omega_{T},
$$

Proof. In view of (21), there exists a subsequence of $\phi_{n}$, not relabeled for convenience, and a function $\phi \in$ $L^{\infty}\left(0, T ; W^{1, \infty}(\Omega)\right)$ such that

$$
\phi_{n} \rightarrow \phi \text { weakly in } L^{q}\left(0, T ; H^{1}(\Omega)\right), v_{n} \rightarrow v=-\kappa \nabla \phi \text { weakly in }\left(L^{q}\left(0, T ;\left(L^{2}(\Omega)\right)\right)^{N}, \forall q \geq 1 .\right.
$$

Let us prove that $\nabla \phi_{n}$ actually strongly converges to $\nabla \phi$.

If $\alpha_{\epsilon_{n}}=0$ for any $n \geq n_{0}, n_{0} \in \mathbb{N}^{*}$, which means that source term $\epsilon_{n} p_{n}+g$ in (5) does not depend on $n$, then $\phi_{n}=\phi$ does not depend on $n$ for $n \geq n_{0}$ and the result is straightforward. Assume now that for any $n_{0} \in \mathbb{N}^{*}$, 
there exists $n \geq n_{0}$ such that $\alpha_{\epsilon_{n}}=1$. We can pick off a subsequence, labeling it the same for convenience, such that $\alpha_{\epsilon_{n}}=1$ for any $n \in \mathbb{N}^{*}$. We check that $\phi_{n}-\phi$ is a weak solution of the following problem:

$$
-\operatorname{div}\left(\kappa \nabla\left(\phi_{n}-\phi\right)\right)=\epsilon_{n} p_{n}-\epsilon_{0} p_{0} \text { in } \Omega_{T}, \phi_{n}-\phi=0 \text { on } \partial \Omega .
$$

We multiply the latter equation by $\phi_{n}-\phi$ and we integrate by parts over $\Omega_{T}$. Thanks to (11), we write

$$
\kappa_{-} \int_{0}^{T} \int_{\Omega}\left|\nabla\left(\phi_{n}-\phi\right)\right|^{2} d x d t \leq \int_{0}^{T} \int_{\Omega}\left(\epsilon_{n} p_{n}-\epsilon_{0} p_{0}\right)\left(\phi_{n}-\phi\right) d x d t .
$$

Since we only assumed that $p_{n}-p_{0} \rightarrow 0$ weakly in $L^{2}\left(\Omega_{T}\right)$, we can not pass directly to the limit in the righthand side of (31). Nevertheless we know, because of the definition of $E_{\epsilon_{n}}$, that $\partial_{t}\left(\epsilon_{n} p_{n}-\epsilon_{0} p_{0}\right)$ is uniformly bounded in $L^{1}(0, T ; X)$. Since $\partial_{t}\left(\phi_{n}-\phi\right)$ satisfies

$$
\left.-\operatorname{div}\left(\kappa \nabla\left(\partial_{t}\left(\phi_{n}-\phi\right)\right)\right)=\partial_{t}\left(\epsilon_{n} p_{n}-\epsilon_{0} p_{0}\right) \text { in } \Omega_{T}, \quad \partial_{t}\left(\phi_{n}-\phi\right)=0 \text { on } \partial \Omega \times\right] 0, T[.
$$

the sequence $\partial_{t}\left(\phi_{n}-\phi\right)$ is also uniformly bounded in $L^{1}(0, T ; X)$. This latter point together with the estimate (21) allows the use of a classical compactness argument of Aubin's type (see e.g. [23] Corollary 4) for ensuring that $\phi_{n}-\phi \rightarrow 0$ in $L^{2}\left(\Omega_{T}\right)$. Then we exploit (31) in the following way:

$$
\kappa_{-} \lim _{n \rightarrow \infty} \int_{0}^{T} \int_{\Omega}\left|\nabla\left(\phi_{n}-\phi\right)\right|^{2} d x d t \leq \lim _{n \rightarrow \infty} \int_{0}^{T} \int_{\Omega}\left(\epsilon_{n} p_{n}-\epsilon_{0} p_{0}\right)\left(\phi_{n}-\phi\right) d x d t=0,
$$

thanks to the strong convergence of $\phi_{n}-\phi$. Convergence result (30) follows.

It follows from (22) that there exists a subsequence, still denoted $c_{n}$ for convenience, and a function $c \in$ $L^{\infty}\left(0, T ; L^{2}(\Omega)\right) \cap L^{2}\left(0, T ; H^{1}(\Omega)\right)$ such that

$$
c_{n} \rightarrow c \text { weakly in } L^{2}\left(0, T ; H^{1}(\Omega)\right) .
$$

Thanks to estimate $(23)$, since $0<\psi_{-} \leq \psi(x) \leq \psi_{+}$a.e. $x \in \Omega$, since $H^{1}(\Omega) \subset L^{2}(\Omega) \subset\left(H^{1}(\Omega)\right)^{\prime}$, the first embedding being compact, we can use an advanced version of Aubin's compactness criterium (see Lemma 3.1 in [12]) to prove that $c_{n}$ is sequentially compact in $L^{p}\left(0, T ; L^{2}(\Omega)\right)$ for any $1 \leq p<2$. Proposition 3.2 is proved.

\section{Generic existence And uniqueness Results}

This section is devoted to the mathematical analysis of the optimal control problems considered in the present paper. First, the question of existence is addressed. Notice that the next lemma both proves the result announced in Theorem 2.3 and the local version of the existence part in Theorem 2.6 (i). We actually introduce a more generic setting by considering the following problem.

Definition 4.1 (Problem $\mathcal{P}_{g e}$ ). Let $T_{g e}>0$. Find $p_{g e}^{*} \in E_{1}$ such that

$$
p_{g e}^{*}=\arg \max _{p \in E_{1}} J(p)=\arg \max _{p \in E_{1}} \int_{0}^{T_{g e}}\left(\int_{\Omega}\left(f(t, x, p)-D\left(x, c_{g e, p}\right)\right) d x\right) e^{-\rho t} d t
$$

where $\left(c_{g e, p}, \phi_{g e, p}\right)$ is a weak solution of the following effective state problem:

$$
\begin{aligned}
& R \psi \partial_{t} c_{g e, p}-\operatorname{div}\left(\psi S\left(v_{g e, p}\right) \nabla c_{g e, p}\right)+v_{g e, p} \cdot \nabla c_{g e, p}=-r\left(c_{g e, p}\right)+p\left(1-c_{g e, p}\right)-g c_{g e, p} \text { in } \Omega \times\left(0, T_{g e}\right), \\
& \operatorname{div}\left(v_{g e, p}\right)=p+g, v_{g e, p}=-\kappa \nabla \phi_{g e, p} \text { in } \Omega \times\left(0, T_{g e}\right), \\
& S\left(v_{g e, p}\right) \nabla c_{g e, p} \cdot n=0 \text { on } \partial \Omega \times\left(0, T_{g e}\right), c_{g e, p \mid t=0}=c_{0} \text { in } \Omega \\
& \phi_{g e, p}=\phi_{1} \text { on } \partial \Omega \times\left(0, T_{g e}\right) .
\end{aligned}
$$


Lemma 4.2. Assume $r$ is a Lipschitz function. For any $T_{g e}>0$, there exists a solution $p_{g e}^{*}$ of Problem $\left(\mathcal{P}_{g e}\right)$. Moreover, $c_{g e}^{*}:=c_{g e, p_{g e}^{*}}$ belongs to $\mathcal{C}\left(\left[0, T_{g e}\right] ; L^{2}(\Omega)\right) \cap L^{2}\left(0, T_{g e} ; H^{1}(\Omega)\right), \phi_{g e}^{*}:=\phi_{g e, p_{g e}^{*}}$ belongs to $L^{\infty}\left(0, T_{g e} ; W^{1, \infty}(\Omega)\right)$. If, moreover, $r(0)=0$ (respectively $\left.r(1)+g \geq 0\right)$ then $c_{g e}^{*} \geq 0$ (respectively $\left.c_{g e}^{*} \leq 1\right)$ almost everywhere in $\Omega \times\left(0, T_{g e}\right)$. In particular, if the latter two conditions are satisfied by $r$, the solution is global, that is the result extents to any $0<T_{g e} \leq \infty$.

Remark 4.3. Notice that the assumptions set for $x \mapsto r(\epsilon, x)$ are sufficient for ensuring that $c_{\epsilon}^{*}$, related to Problem $\mathcal{P}_{\epsilon}$, satisfies $0 \leq c_{\epsilon}^{*} \leq 1 / \epsilon$ a.e. in $\Omega \times\left(0, T_{g e}\right)$, for any $T_{g e}>0$. The global existence of a solution for $\mathcal{P}_{\epsilon}$ follows directly. The definition of $r_{0}$ does not allow the same direct consideration for $\mathcal{P}_{0}$. However the global existence result for $\mathcal{P}_{0}$ will be ensured by the convergence of a sequence of global solutions of $\mathcal{P}_{\epsilon}$ in the asymptotic analysis below (Section 5).

Proof. The existence of a weak solution for the state system (32)-(35), for any given $p \in E_{1}$, satisfying $c_{g e, p} \in$ $\mathcal{C}\left(\left[0, T_{g e}\right] ; L^{2}(\Omega)\right) \cap L^{2}\left(0, T_{g e} ; H^{1}(\Omega)\right), \phi_{g e} \in L^{\infty}\left(0, T_{g e} ; W^{1, \infty}(\Omega)\right)$ and $c_{g e, p} \geq 0$ a.e. if $r(0)=0$ (respectively $c_{g e, p} \leq 1$ if $r(1)+g \geq 0$ ) is a direct consequence of Proposition 2.2 (which is proved in [1]). It follows in particular that the set

$$
\left\{J(p) ; p \in E_{1}\right\} \subset \mathbb{R}
$$

is nonempty. Since any $p \in E_{1}$ satisfies $0 \leq p(t, x) \leq \bar{p}$ almost everywhere in $\Omega \times\left(0, T_{g e}\right)$, and thanks to the assumptions on $f$ and $D$, we assert that the latter set is also a bounded set of $\mathbb{R}$. Thus, it admits a supremum, denoted by $J^{*}$. Our aim is to prove that there exists $p_{g e}^{*}$ such that

$$
J^{*}=J\left(p_{g e}^{*}\right),
$$

meaning that the supremum is actually a maximum.

By definition of the supremum, there exists a sequence $\left(p_{n}\right)_{n \in \mathbf{N}} \subset E_{1}$, associated to the sequence $\left(c_{g e, p_{n}}:=\right.$ $\left.c_{n}, \phi_{g e, p_{n}}:=\phi_{n}\right)_{n \in \mathbf{N}}$ of solutions to the state problem, such that

$$
\lim _{n \rightarrow \infty} J\left(p_{n}\right)=J^{*}
$$

The stability results of Proposition 3.2 remain true in this generic setting (here $\epsilon_{n}=\epsilon$ for any $n$ ). Thus, there exists $\left(p_{*}, c_{*}, \phi_{*}\right) \in L^{2}\left(\Omega \times\left(0, T_{g e}\right)\right) \times L^{2}\left(0, T_{g e} ; H^{1}(\Omega) \times L^{\infty}\left(0, T_{g e} ; W^{1, \infty}(\Omega)\right)\right.$ and a subsequence, non renamed for convenience, such that, for any $u \geq 1$,

$$
\begin{aligned}
& c_{n} \rightarrow c_{*} \text { weakly in } L^{2}\left(0, T_{g e} ; H^{1}(\Omega)\right), c_{n} \rightarrow c \text { in } L^{s}\left(0, T_{g e} ; L^{2}(\Omega)\right), 1 \leq s<2, \text { and a.e. in } \Omega_{T}, \\
& \phi_{n} \rightarrow \phi_{*} \text { weakly in } L^{u}\left(0, T_{g e} ; W^{1, u}(\Omega)\right), \\
& v_{n} \rightarrow v_{*}=-\kappa \nabla \phi_{*} \text { in }\left(L^{2}\left(\Omega \times\left(0, T_{g e}\right)\right)\right)^{N} \text { and a.e. in } \Omega \times\left(0, T_{g e}\right) .
\end{aligned}
$$

These convergence results are sufficient to pass to the limit in the state problem,

$$
\begin{aligned}
& R \psi \partial_{t} c_{n}+v_{n} \cdot \nabla c_{n}-\operatorname{div}\left(\psi S\left(v_{n}\right) \nabla c_{n}\right)=-r\left(c_{n}\right)+p_{n}\left(1-c_{n}\right)-g c_{n} \text { in } \Omega \times\left(0, T_{g e}\right), \\
& \operatorname{div}\left(v_{n}\right)=p_{n}+g, v_{n}=-\kappa \nabla \phi_{n} \text { in } \Omega \times\left(0, T_{g e}\right), \\
& S\left(v_{n}\right) \nabla c_{n} \cdot n=0 \text { on } \partial \Omega \times\left(0, T_{g e}\right), c_{n \mid t=0}=c_{0} \text { in } \Omega, \phi_{n}=\phi_{1} \text { on } \partial \Omega \times\left(0, T_{g e}\right),
\end{aligned}
$$

using also Lebesgue's dominated convergence theorem for nonlinear terms and the continuity of the function $c \in \mathcal{C}\left(0, T_{g e} ; \Omega\right) \mapsto c_{\mid t=0}$ for the initial condition. We conclude that the limit $\left(c_{*}, \phi_{*}\right)$ of $\left(c_{n}, \phi_{n}\right)$ is a weak solution of the state equation associated to $p_{*}$, meaning $c_{*}=c_{g e, p_{*}}, \phi_{*}=\phi_{g e, p_{*}}$. Moreover, the maximum principle for $c_{*}$ is preserved when $r$ satisfies the corresponding assumptions.

It remains to show that

$$
\lim _{n \rightarrow \infty} J\left(p_{n}\right)=J\left(p_{*}\right) .
$$


Indeed, since $J^{*}$ is defined by $\lim _{n \rightarrow \infty} J\left(p_{n}\right)=J^{*}$, the proof will be ended. The assumptions making $D$ a $M$-type operator let us claim that

$$
\lim _{n \rightarrow \infty} \int_{0}^{T_{g e}}\left(\int_{\Omega} D\left(x, c_{n}\right) d x\right) e^{-\rho t} d t=\int_{0}^{T_{g e}}\left(\int_{\Omega} D\left(x, c_{*}\right) d x\right) e^{-\rho t} d t .
$$

Then

$$
\begin{aligned}
J^{*} & =\lim _{n \rightarrow \infty} \int_{0}^{T_{g e}}\left(\int_{\Omega}\left(f\left(t, x, p_{n}\right)-D\left(x, c_{n}\right)\right) d x\right) e^{-\rho t} d t \\
& =\lim _{n \rightarrow \infty} \int_{0}^{T_{g e}}\left(\int_{\Omega} f\left(t, x, p_{n}\right) d x\right) e^{-\rho t} d t-\int_{0}^{T_{g e}}\left(\int_{\Omega} D\left(x, c_{*}\right) d x\right) e^{-\rho t} d t \\
& =\limsup _{n \rightarrow \infty} \int_{0}^{T_{g e}}\left(\int_{\Omega} f\left(t, x, p_{n}\right) d x\right) e^{-\rho t} d t-\int_{0}^{T_{g e}}\left(\int_{\Omega} D\left(x, c_{*}\right) d x\right) e^{-\rho t} d t .
\end{aligned}
$$

Using $p_{n} \rightarrow p_{*}$ in $L^{2}\left(\Omega \times\left(0, T_{g e}\right)\right)$, the concavity of $f$ and the lower semi-continuity of concave functions (Tartar [24]), we obtain

$$
\limsup _{n \rightarrow \infty} \int_{0}^{T_{g e}}\left(\int_{\Omega} f\left(t, x, p_{n}\right) d x\right) e^{-\rho t} d t \leq \int_{0}^{T_{g e}}\left(\int_{\Omega} f\left(t, x, p_{*}\right) d x\right) e^{-\rho t} d t .
$$

We conclude from the two latter relations that

$$
J^{*} \leq \int_{0}^{T_{g e}}\left(\int_{\Omega}\left(f\left(t, x, p_{*}\right)-D\left(x, c_{*}\right)\right) d x\right) e^{-\rho t} d t=J\left(p_{*}\right) .
$$

However, $p_{*} \in E_{1}$. Indeed, since $\left\|p_{n}\right\|_{L^{\infty}\left(\Omega \times\left(0, T_{g e}\right)\right)} \leq \bar{p}$ for any $n \in \mathbb{N}, p_{n}$ also tends to $p_{*}$ in $L^{\infty}\left(\Omega \times\left(0, T_{g e}\right)\right)$ weak-* and $\left\|p_{*}\right\|_{L^{\infty}\left(\Omega \times\left(0, T_{g e}\right)\right)} \leq \liminf \left\|p_{n}\right\|_{L^{\infty}\left(\Omega \times\left(0, T_{g e}\right)\right)} \leq \bar{p}$. Likewise, $\left\|p_{*}-\bar{p}\right\|_{L^{\infty}\left(\Omega \times\left(0, T_{g e}\right)\right)} \leq \bar{p}$. It follows that $0 \leq p_{*}(t, x) \leq \bar{p}$ almost everywhere in $\Omega \times\left(0, T_{g e}\right)$. Since $p_{*} \in E_{1}$ and $c_{*}=c_{g e, p_{*}}$, by definition of the supremum $J^{*}$, we have

$$
J\left(p_{*}\right) \leq J^{*} .
$$

In view of the two latter relations, $J\left(p_{*}\right)=J^{*}$. This ends the proof of the lemma.

We now state and prove a well-posedness result for Problem $\mathcal{P}_{0}$. The following lemma is a second step in the proof of Theorem 2.6 (i).

Lemma 4.4. There exists a unique optimal solution $p^{*}$ of Problem $\mathcal{P}_{0}$ given in Definition 2.5.

Proof. The existence of an optimal solution $p^{*}$ is obtained by Lemma 4.2. Besides, the asymptotic analysis performed in Section 5 below will furnish another proof of the existence result for $\mathcal{P}_{0}$ and will ensure its global character. It remains to prove uniqueness. Let us do it by contradiction. Let $p_{1}^{*}$ and $p_{2}^{*}$ be two optimal solutions of $\mathcal{P}_{0}$, with $p_{1}^{*} \neq p_{2}^{*}$ almost everywhere in $\Omega_{T}$. They are associated with $c_{p_{i}^{*}}:=c_{i}^{*}, i=1,2$, satisfying (17)-(20) with $p=p_{i}^{*}$. We denote by $M_{0}$ the value of the objective for the optimal solution:

$$
M_{0}:=J\left(p_{1}^{*}\right)=J\left(p_{2}^{*}\right)
$$

We aim at comparing $M_{0}$ with $J\left(\left(p_{1}^{*}+p_{2}^{*}\right) / 2\right)$.

By definition of $J$,

$$
J\left(\frac{1}{2}\left(p_{1}^{*}+p_{2}^{*}\right)\right)=\int_{0}^{T}\left(\int_{\Omega}\left(f\left(t, x, \frac{1}{2} p_{1}^{*}+\frac{1}{2} p_{2}^{*}\right)-D\left(x, c^{\prime}\right)\right) d x\right) e^{-\rho t} d t
$$


where $c^{\prime}=c_{\left(p_{1}^{*}+p_{2}^{*}\right) / 2}$ is a weak solution of solution of

$$
\begin{aligned}
& R \psi \partial_{t} c^{\prime}+v \cdot \nabla c^{\prime}-\operatorname{div}\left(\psi S(v) \nabla c^{\prime}\right)=-r_{0}\left(c^{\prime}\right)-g c^{\prime}+\left(p_{1}^{*}+p_{2}^{*}\right) / 2 \text { in } \Omega_{T}, \\
& S(v) \nabla c^{\prime} \cdot n=0 \text { on } \partial \Omega \times(0, T), c^{\prime}{ }_{\mid t=0}=c_{0} \text { in } \Omega .
\end{aligned}
$$

Due to the strict concavity of $f$, we have

$$
J\left(\frac{1}{2}\left(p_{1}^{*}+p_{2}^{*}\right)\right)>\int_{0}^{T}\left(\int_{\Omega}\left(\frac{1}{2} f\left(t, x, p_{1}^{*}\right)+\frac{1}{2} f\left(t, x, p_{2}^{*}\right)-D\left(x, c^{\prime}\right)\right) d x\right) e^{-\rho t} d t .
$$

We now compare $D\left(x, c^{\prime}\right)$ and $D(x, c)$ where $c=\left(c_{1}^{*}+c_{2}^{*}\right) / 2$. We infer from (17)-(20) that $c$ is a weak solution of

$$
\begin{aligned}
& R \psi \partial_{t} c+v \cdot \nabla c-\operatorname{div}(\psi S(v) \nabla c)=-\left(r_{0}\left(c_{1}^{*}\right)+r_{0}\left(c_{2}^{*}\right)\right) / 2-g c+\left(p_{1}^{*}+p_{2}^{*}\right) / 2 \text { in } \Omega_{T} \\
& S(v) \nabla c \cdot n=0 \text { on } \partial \Omega \times(0, T), c_{\mid t=0}=c_{0} \text { in } \Omega .
\end{aligned}
$$

Let $d=c-c^{\prime}$. Let us show that $d \geq 0$ almost everywhere in $\Omega_{T}$. In view of (36) and (38), $d$ satisfies the following equations:

$$
\begin{aligned}
& R \psi \partial_{t} d+v \cdot \nabla d-\operatorname{div}(\psi S(v) \nabla d)=-\left(r_{0}\left(c_{1}^{*}\right)+r_{0}\left(c_{2}^{*}\right)\right) / 2+r_{0}\left(c^{\prime}\right)-g d \text { in } \Omega_{T} \\
& S(v) \nabla d \cdot n=0 \text { on } \partial \Omega \times(0, T), d_{\mid t=0}=0 \text { in } \Omega
\end{aligned}
$$

The trick consists in rewriting the first equation in the form

$$
R \psi \partial_{t} d+v \cdot \nabla d-\operatorname{div}(\psi S(v) \nabla d)=r_{0}\left(\frac{1}{2}\left(c_{1}^{*}+c_{2}^{*}\right)\right)-\frac{r_{0}\left(c_{1}^{*}\right)}{2}-\frac{r_{0}\left(c_{2}^{*}\right)}{2}-\left(r_{0}\left(\frac{1}{2}\left(c_{1}^{*}+c_{2}^{*}\right)\right)-r_{0}\left(c^{\prime}\right)\right)-g d
$$

We multiply this equation by $d^{-}=\min (0, d)$ and we integrate by parts over $\Omega \times(0, \tau), \tau>0$. We get

$$
\begin{aligned}
\frac{R}{2} \int_{0}^{\tau} \frac{d}{d t} \int_{\Omega} & \psi\left|d^{-}\right|^{2} d x d t+\int_{0}^{\tau} \int_{\Omega} \psi S(v) \nabla d \cdot \nabla d^{-} d x d t=-\int_{0}^{\tau} g\left|d^{-}\right|^{2} d x d t \\
& +\int_{0}^{\tau} \int_{\Omega}\left(r_{0}\left(\frac{1}{2}\left(c_{1}^{*}+c_{2}^{*}\right)\right)-\frac{r_{0}\left(c_{1}^{*}\right)}{2}-\frac{r_{0}\left(c_{2}^{*}\right)}{2}\right) d^{-} d x d t \\
& -\int_{0}^{\tau} \int_{\Omega}\left(r_{0}\left(\frac{1}{2}\left(c_{1}^{*}+c_{2}^{*}\right)\right)-r_{0}\left(c^{\prime}\right)\right) d^{-} d x d t+\int_{0}^{\tau} \int_{\Omega}(v \cdot \nabla d) d^{-} d x d t
\end{aligned}
$$

We know that

$$
\int_{\Omega} \psi\left|d^{-}\right|^{2} d x \geq \int_{\Omega} \psi_{-} \int_{\Omega}\left|d^{-}\right|^{2} d x, \quad \int_{\Omega} \psi S(v) \nabla d \cdot \nabla d^{-} d x \geq \psi_{-} S_{m} \int_{\Omega}\left|\nabla d^{-}\right|^{2} d x .
$$

Using Cauchy-Schwarz and Young inequalities, we compute

$$
\left|\int_{0}^{\tau} \int_{\Omega}(v \cdot \nabla d) d^{-} d x d t\right| \leq \frac{\psi_{-} S_{m}}{2} \int_{0}^{\tau} \int_{\Omega}\left|\nabla d^{-}\right|^{2} d x d t+C\|v\|_{L^{\infty}\left(\Omega_{T}\right)} \int_{0}^{\tau} \int_{\Omega}\left|d^{-}\right|^{2} d x d t
$$

Using moreover the derivability of $r_{0}$, relation (40) gives

$$
\begin{array}{r}
\frac{R \psi_{-}}{2} \int_{0}^{\tau} \frac{d}{d t} \int_{\Omega}\left|d^{-}\right|^{2} d x d t+\frac{\psi_{-} S_{m}}{2} \int_{0}^{\tau} \int_{\Omega}\left|\nabla d^{-}\right|^{2} d x d t-\int_{0}^{\tau} \int_{\Omega}\left(r_{0}\left(\frac{1}{2}\left(c_{1}^{*}+c_{2}^{*}\right)\right)-\frac{r_{0}\left(c_{1}^{*}\right)}{2}-\frac{r_{0}\left(c_{2}^{*}\right)}{2}\right) d^{-} d x d t \\
\leq\left(\|g\|_{L^{\infty}\left(\Omega_{T}\right)}+\left\|r_{0}^{\prime}\right\|_{L^{\infty}(\mathbb{R})}+C\|v\|_{L^{\infty}\left(\Omega_{T}\right)}\right) \int_{0}^{\tau} \int_{\Omega}\left|d^{-}\right|^{2} d x d t .
\end{array}
$$


The third term in the lefthand side of the latter relation is nonnegative because function $r_{0}$ is concave. Using Gronwall's Lemma, we thus get, for any $\tau \in(0, T)$,

$$
\int_{0}^{\tau} \int_{\Omega}\left|d^{-}\right|^{2} d x d t \leq 0
$$

Then $d^{-}=0$ and $d=c-c^{\prime} \geq 0$ almost everywhere in $\Omega_{T}$.

Remind that our aim was to compare $D\left(\cdot, c^{\prime}\right)$ and $D(\cdot, c)$. Since $D$ is an increasing function, we infer from $c-c^{\prime} \geq 0$ that $D(\cdot, c) \geq D\left(\cdot, c^{\prime}\right)$ almost everywhere in $\Omega_{T}$. Then, due to (37) and to the convexity of $c \mapsto D(\cdot, c)$, we have

$$
\begin{aligned}
J\left(\frac{1}{2}\left(p_{1}^{*}+p_{2}^{*}\right)\right) & >\int_{0}^{T}\left(\int_{\Omega} \frac{1}{2} f\left(t, x, p_{1}\right)+\frac{1}{2} f\left(x, p_{2}\right)-D\left(x, c^{\prime}\right) d x\right) e^{-\rho t} d t \\
& \geq \int_{0}^{T}\left(\int_{\Omega} \frac{1}{2} f\left(t, x, p_{1}^{*}\right)+\frac{1}{2} f\left(t, x, p_{2}^{*}\right)-D(x, c) d x\right) e^{-\rho t} d t \\
& =\int_{0}^{T}\left(\int_{\Omega} \frac{1}{2} f\left(t, x, p_{1}^{*}\right) d x+\int_{\Omega} \frac{1}{2} f\left(t, x, p_{2}^{*}\right) d x-\int_{\Omega} D\left(x, \frac{1}{2} c_{1}^{*}+\frac{1}{2} c_{2}^{*}\right) d x\right) e^{-\rho t} d t \\
& \geq \frac{1}{2} \int_{0}^{T}\left(\int_{\Omega} f\left(t, x, p_{1}^{*}\right) d x-\int_{\Omega} D\left(x, c_{1}^{*}\right) d x+\int_{\Omega} f\left(t, x, p_{2}^{*}\right) d x-\int_{\Omega} D\left(x, c_{2}^{*}\right) d x\right) e^{-\rho t} d t \\
& =2 \times \frac{1}{2} M_{0}
\end{aligned}
$$

that is $J\left(\left(p_{1}+p_{2}\right) / 2\right)>M_{0}$. There is a contradiction with the definition of the maximal value $M_{0}$. We have proved the uniqueness of the solution of the problem $\mathcal{P}_{0}$.

\section{Asymptotic Analysis}

In this section, we prove that any sequence of optimal solutions $p_{\epsilon}^{*}$ of the scaled problem $\mathcal{P}_{\epsilon}$ tends, as $\epsilon \rightarrow 0$, to the unique solution $p$ of the upscaled problem $\mathcal{P}_{0}$. Thus, all at once, we derive rigorously the upscaled optimal control problem for low concentrations and we prove that this effective problem is globally well-posed. This result, though already stated in Theorem 2.6 , is recalled below.

Lemma 5.1. As $\epsilon \rightarrow 0$, any sequence of optimal solutions $\left(p_{\epsilon}^{*}, c_{\epsilon}^{*}, \phi_{\epsilon}^{*}\right)$ of Problem $\mathcal{P}_{\epsilon}$ in the sense of Definitions 1.1 and 2.1 converges to the unique optimal solution $\left(p^{*}, c^{*}, \phi\right)$ of Problem $\mathcal{P}_{0}$ defined by Definition 2.5:

$$
\begin{aligned}
& p_{\epsilon}^{*} \rightarrow p^{*} \text { weakly in } L^{u}\left(\Omega_{T}\right), \forall u \geq 1, \\
& \phi_{\epsilon}^{*} \rightarrow \phi \text { weakly in } L^{u}\left(0, T ; W^{1, u}(\Omega)\right), \forall u \geq 1, \\
& v_{\epsilon}^{*} \rightarrow v=-\kappa \nabla \phi \text { in }\left(L^{2}\left(\Omega_{T}\right)\right)^{N} \text { and a.e. in } \Omega_{T}, \\
& c_{\epsilon}^{*} \rightarrow c^{*} \text { weakly in } L^{2}\left(0, T ; H^{1}(\Omega)\right), c_{\epsilon}^{*} \rightarrow c^{*} \text { in } L^{s}\left(0, T ; L^{2}(\Omega)\right), 1 \leq s<2, \text { and a.e. in } \Omega_{T} .
\end{aligned}
$$

Let us add a remark about the sets of admissible controls. As $\epsilon \rightarrow 0, E_{\epsilon}$ tends to $E_{0}$ because of the weighting by $\epsilon$ in the condition involving $L^{1}(0, T ; X)$. Of course, for $\epsilon>0$, mentioning this weighting was useless. However we have chosen to add it for emphasizing that the constraint in $L^{1}(0, T ; X)$ disappears as $\epsilon \rightarrow 0$, which is natural since the limit hydraulic pressure equation does not depend on the pollutant load $p$.

Proof. Let $\left(p_{\epsilon}^{*}, c_{\epsilon}^{*}, \phi_{\epsilon}^{*}\right)$ a sequence of optimal solutions of Problem $\mathcal{P}_{\epsilon}$. Since $0 \leq p_{\epsilon}^{*} \leq \bar{p}$ almost everywhere in $\Omega_{T}$, the sequence $\left(p_{\epsilon}^{*}\right)$ is uniformly bounded in $L^{\infty}\left(\Omega_{T}\right)$. Then there exists a subsequence, denoted the same for simplicity, and a function $p \in E_{0}$ such that

$$
p_{\epsilon}^{*} \stackrel{*}{\rightarrow} p \text { weakly-* in } L^{\infty}\left(\Omega_{T}\right) .
$$


Since Proposition 3.2 applies, we also assert that there exist $(c, \phi) \in L^{2}\left(0, T ; H^{1}(\Omega)\right) \times L^{\infty}\left(0, T ; W^{1, \infty}(\Omega)\right)$ and a subsequence, not renamed for convenience, such that

$$
\begin{aligned}
& c_{\epsilon}^{*} \rightarrow c \text { weakly in } L^{2}\left(0, T ; H^{1}(\Omega)\right), c_{\epsilon}^{*} \rightarrow c \text { in } L^{s}\left(0, T ; L^{2}(\Omega)\right), 1 \leq s<2, \text { and a.e. in } \Omega_{T}, \\
& \phi_{\epsilon}^{*} \rightarrow \phi \text { weakly in } L^{u}\left(0, T ; W^{1, u}(\Omega)\right), \forall u \geq 1, \\
& v_{\epsilon}^{*} \rightarrow v=-\kappa \nabla \phi \text { in }\left(L^{2}\left(\Omega_{T}\right)\right)^{N} \text { and a.e. in } \Omega_{T} .
\end{aligned}
$$

These results are sufficient to derive the effective state system. More precisely, using Lebesgue's dominated convergence theorem for nonlinear terms and the continuity of function $c \in \mathcal{C}(0, T ; \Omega) \mapsto c_{\mid t=0}$ for the initial condition, letting $\epsilon \rightarrow 0$ in the variational formulation of

$$
\begin{aligned}
& \operatorname{div}\left(v_{\epsilon}^{*}\right)=\epsilon p_{\epsilon}^{*}+g, v_{\epsilon}^{*}=-\kappa \nabla \phi_{\epsilon}^{*} \text { in } \Omega_{T}, \phi_{\epsilon}^{*}=\phi_{1} \text { on } \partial \Omega \times(0, T), \\
& R \psi \partial_{t} c_{\epsilon}^{*}+v_{\epsilon}^{*} \cdot \nabla c_{\epsilon}^{*}-\operatorname{div}\left(\psi S\left(v_{\epsilon}^{*}\right) \nabla c_{\epsilon}^{*}\right)=-r_{\epsilon}\left(c_{\epsilon}^{*}\right)-g c_{\epsilon}^{*}+p_{\epsilon}^{*}-\epsilon p_{\epsilon}^{*} c_{\epsilon}^{*} \text { in } \Omega_{T}, \\
& S\left(v_{\epsilon}^{*}\right) \nabla c_{\epsilon}^{*} \cdot n=0 \text { on } \partial \Omega \times(0, T), c_{\epsilon_{\mid t=0}^{*}}^{*} \text { in } \Omega,
\end{aligned}
$$

bearing in mind the pointwise convergence of $r_{\epsilon}$ to the continuous function $r_{0}$, we obtain the variational formulation of

$$
\begin{aligned}
& \operatorname{div}(v)=g, v=-\kappa \nabla \phi \text { in } \Omega_{T}, \phi=\phi_{1} \text { on } \partial \Omega \times(0, T), \\
& R \psi \partial_{t} c+v \cdot \nabla c-\operatorname{div}(\psi S(v) \nabla c)=-r_{0}(c)-g c+p \text { in } \Omega_{T}, \\
& S(v) \nabla c \cdot n=0 \text { on } \partial \Omega \times(0, T), c_{\mid t=0} \text { in } \Omega .
\end{aligned}
$$

It means actually that $(c, \phi)=\left(c_{p}, \phi_{p}\right)$ defined by (17)-(20). The effective state system is derived.

We now study the limit behavior of the optimization problem. Let $M_{\epsilon}$ (resp. $M_{0}$ ) be the maximal value of the objective for Problem $\mathcal{P}_{\epsilon}\left(\right.$ resp. $\left.\mathcal{P}_{0}\right)$, that is

$$
\begin{aligned}
& M_{\epsilon}=J\left(p_{\epsilon}^{*}\right)=\int_{0}^{T}\left(\int_{\Omega} f\left(t, x, p_{\epsilon}^{*}\right)-D\left(x, c_{\epsilon}^{*}\right) d x\right) e^{-\rho t} d t \\
& M_{0}=J\left(p^{*}\right)=\int_{0}^{T}\left(\int_{\Omega} f\left(t, x, p^{*}\right)-D\left(x, c^{*}\right) d x\right) e^{-\rho t} d t=\max _{q \in E_{0}} \int_{0}^{T}\left(\int_{\Omega} f(t, x, q)-D\left(x, c_{q}\right) d x\right) e^{-\rho t} d t .
\end{aligned}
$$

We aim at proving that $p=p^{*}$. In view of the uniqueness result of Lemma 4.4, it is sufficient for our purpose to prove that $J(p)=M_{0}$. Of course, since $p \in E_{0}$, we only know that

$$
J(p) \leq M_{0}
$$

Let us sketch the main steps of the remainder of the proof:

Step 0 We know that $J(p) \leq M_{0}$.

Step 1 We use the structure of the objective $J$ for proving that $\lim \sup _{\epsilon \rightarrow 0} M_{\epsilon} \leq J(p)$.

Step 2 We construct a perturbation of the optimal state concentration $c^{*}=c_{p^{*}}$, actually $c_{\epsilon, p^{*}}$, and we prove that $M_{0} \leq \liminf _{\epsilon \rightarrow 0} M_{\epsilon}$.

Step 3 We conclude that $J(p)=M_{0}$.

Step 1 is based on classical continuity type arguments. Using the strong convergence of $c_{\epsilon}^{*}$ and the assumptions on $D$, we claim with a monotonicity argument that

$$
-\int_{0}^{T} \int_{\Omega} D(x, c) e^{-\rho t} d x d t=\lim _{\epsilon \rightarrow 0}\left(-\int_{0}^{T} \int_{\Omega} D\left(x, c_{\epsilon}^{*}\right) e^{-\rho t} d x d t\right) .
$$


Since $f$ is concave and $p_{\epsilon}^{*} \stackrel{*}{\rightarrow} p$ weakly-* in $L^{\infty}\left(\Omega_{T}\right)$, we know, according to the inferior lower-semicontinuity of concave functions theorem, that

$$
\int_{0}^{T} \int_{\Omega} f(t, x, p) e^{-\rho t} d x d t \geq \limsup _{\epsilon \rightarrow 0} \int_{0}^{T} \int_{\Omega} f\left(t, x, p_{\epsilon}^{*}\right) e^{-\rho t} d x d t .
$$

Thus

$$
J(p)=\int_{0}^{T}\left(\int_{\Omega} f(t, x, p)-D(x, c) d x\right) e^{-\rho t} d t \geq \limsup _{\epsilon \rightarrow 0} M_{\epsilon} .
$$

In Step 2, we look for an appropriate approximation of $c^{*}$. This point is the tricky part of the proof. For the convenience of the reader, we recall that $c^{*}$ is a weak solution of

$$
\left\{\begin{array}{l}
R \psi \partial_{t} c^{*}+v \cdot \nabla c^{*}-\operatorname{div}\left(\psi S(v) \nabla c^{*}\right)=-r_{0}\left(c^{*}\right)-g c^{*}+p^{*} \text { in } \Omega_{T}, \\
\operatorname{div}(v)=g, v=-\kappa \nabla \phi \text { in } \Omega_{T}, \\
S(v) \nabla c^{*} \cdot n=0 \text { on } \partial \Omega \times(0, T), c_{\mid t=0}^{*}=c_{0} \text { in } \Omega, \phi=\phi_{1} \text { in } \partial \Omega \times(0, T) .
\end{array}\right.
$$

Since $v$ does not depend on $c^{*}$, Proposition 3.3. in [1] ensures that $c^{*}$ is actually uniquely defined by (43). Let us now consider $c_{\epsilon, p^{*}}$, which is defined by (4)-(5) with $p=p^{*}$ and (7)-(9), that is:

$$
\left\{\begin{array}{l}
R \psi \partial_{t} c_{\epsilon, p^{*}}+v_{\epsilon, p^{*}} \cdot \nabla c_{\epsilon, p^{*}}-\operatorname{div}\left(\psi S\left(v_{\epsilon, p^{*}}\right) \nabla c_{\epsilon, p^{*}}\right)=-r_{\epsilon}\left(c_{\epsilon, p^{*}}\right)-g c_{\epsilon, p^{*}}+p^{*}\left(1-\epsilon c_{\epsilon, p^{*}}\right) \text { in } \Omega_{T}, \\
\operatorname{div}\left(v_{\epsilon, p^{*}}\right)=\epsilon p^{*}+g, v_{\epsilon, p^{*}}=-\kappa \nabla \phi_{\epsilon, p^{*}} \text { in } \Omega_{T}, \\
S\left(v_{\epsilon, p^{*}}\right) \nabla c_{\epsilon, p^{*}} \cdot n=0 \text { on } \partial \Omega \times(0, T), c_{\epsilon, p^{*} \mid t=0}=c_{0}^{*} \text { in } \Omega, \phi_{\epsilon, p^{*}}=\phi_{1} \text { on } \partial \Omega \times(0, T)
\end{array}\right.
$$

Once again, we exploit Proposition 3.2 (in the case $p_{n}=p^{*}$ for any $n$ ) and we study the behavior of (44) as $\epsilon \rightarrow 0$. In particular $c_{\epsilon, p^{*}}$ is sequentially compact in $L^{2}\left(\Omega_{T}\right)$. There exists a subsequence of $c_{\epsilon, p^{*}}$ converging in $L^{2}\left(\Omega_{T}\right)$ to a function, which turns out to be a weak solution of (43). The solution of this latter problem being unique, the whole sequence converges and the limit is

$$
c_{\epsilon, p^{*}} \rightarrow c^{*} \text { in } L^{2}\left(\Omega_{T}\right) \text { and a.e. in } \Omega_{T} .
$$

Moreover

$$
\begin{aligned}
M_{0}=\int_{0}^{T} & \left(\int_{\Omega} f\left(t, x, p^{*}\right)-D\left(x, c^{*}\right) d x\right) e^{-\rho t} d t \\
& =\lim _{\epsilon \rightarrow 0} \int_{0}^{T}\left(\int_{\Omega} f\left(t, x, p^{*}\right)-D\left(x, c_{\epsilon, p^{*}}\right) d x\right) e^{-\rho t} d t \leq \liminf _{\epsilon \rightarrow 0} M_{\epsilon},
\end{aligned}
$$

the second equality being justified by the convergence of $c_{\epsilon, p^{*}}$ and a monotonicity argument, the inequality being justified by the definition of the maximal value $M_{\epsilon}$.

Summing up the results in Step 3, with (41), (42) and (45), we have

$$
M_{0} \leq \liminf _{\epsilon \rightarrow 0} M_{\epsilon} \leq \limsup _{\epsilon \rightarrow 0} M_{\epsilon} \leq J(p) \leq M_{0}
$$

and thus

$$
J(p)=M_{0} .
$$

In view of the uniqueness result of Lemma 4.4, it follows that $p=p^{*}$ and that the whole sequence $\left(p_{\epsilon}^{*}, c_{\epsilon}^{*}, \phi_{\epsilon}^{*}\right)$ converges to $\left(p^{*}, c^{*}, \phi\right)$. This ends the proof of the lemma. 
Theorem 2.6 is proved by Lemmas 4.4 and 5.1.

\section{Conclusion}

This paper presents a realistic model that computes the optimal trade-off between the use of a polluting process for gaining productivity and the cleaning treatment cost for supplying the population with drinking water. The hydrogeological model for the spread of the pollutant in the groundwater is space dependent. The economic cost both depends on the pollution degree and on the treatment plants location. An existence result of an optimal solution is provided in a generic setting. Since the pollutant concentration in groundwater is usually small, we justify the use of asymptotic analysis tools for further proving the uniqueness of the optimal control. Notice that the convergence of the optimal solution of the scaled problem to the optimal solution of the effective one is also obtained, which is not a foreseeable result.

At least two interesting extensions of the present model could be explored. First, we have considered here an optimal control problem that can be solved by a central planner. However, the pollution may be issued from several sources (for instance, agricultural pollution is due to distinct farmers). These agents may differ, for instance, from their distance to the treatment plant and/or from their productivity gains related to the use of the polluting process. The framework we consider here has thus also to be studied from a game theory point of view. Moreover, beyond the existence of the game equilibrium, the qualitative comparison of the game solution with the optimal control solution could be interesting from both a theoretical and a assessment point of view. Second, we did not assume the existence of buffer zones between the polluted area and the treatment plant. Experimental data show that active buffer zones may contribute to the cleaning of soils and groundwater from contaminants. An ecosystem dynamics providing a cleaning service could be included in the present model. It would be then worth computing the value of this ecosystem service.

\section{REFERENCES}

[1] E. Augeraud-Véron, C. Choquet and É. Comte, Optimal control for a groundwater pollution ruled by a convection-diffusionreaction problem. Journal of Optimization Theory Applications 173(3) (2017) 941-966, https://doi.org/10.1007/s10957-016-10178

[2] E. Augeraud-Véron and M. Leandri, Optimal pollution control with distributed delays. Journal of Mathematical Economics 55 (2014) 24-32, http://dx.doi.org/10.1016/j.jmateco.2014.09.010.

[3] J . Bear and A. Verruijt, Modeling Groundwater Flow and Pollution. Theory and Applications of Transport in Porous Media (1987).

[4] C. Benosman, B. Ainseba and A. Ducrot, Optimization of cytostatic leukemia therapy in an advection reaction diffusion model. Journal of Optimization Theory Applications 167 (2015) 296-325, http://dx.doi.org/10.1007/s10957-014-0667-7.

[5] P. Bordenave, F. Bouraoui, C. Gascuel-Odoux, J. Molénat and P. Mérot, Décalages temporels entre des modifications des pratiques agricoles et la diminution des nitrates dans les eaux superficielles, in Merceron, M. (Ed), Pollutions diffuses : du bassin au littoral (IFREMER) Ploufargan (1999), 311-333.

[6] W. Brock and A. Xepapadeas, Diffusion-induced instability and pattern formation in infinite horizon recursive optimal control. Journal of Economic Dynamics and Control 32 (2008) 2745-2787, http://dx.doi.org/10.2139/ssrn.895682.

[7] C. Camacho and A. Pérez-Barahona, Land use dynamics and the environment. Journal of Economic Dynamics and Control 52 (2015) 96-118, https://doi.org/10.1016/j.jedc.2014.11.013.

[8] C. Choquet and A. Mikelić, Rigorous upscaling of the reactive flow with finite kinetics and under dominant Péclet number. Continuum Mechanics and Thermodynamics 21 (2009) 125-140, http://dx.doi.org/10.1007/s00161-009-0099-z.

[9] R. M. Clark and P. Dorsey, A model of costs for treating drinking water. Journal (American Water Works Association) 74(12) (1982) 618-627. https://doi.org/10.1002/j.1551-8833.1982.tb05027.x.

[10] D. Dearmont, B. A. McCarl and D. A. Tolman, Costs of water treatment due to diminished water quality: a case study in Texas. Water Resources Research 34.4 (1998) 849-853. https://doi.org/10.1029/98WR00213.

[11] J. de Frutos and G. Martin-Herran, Spatial effects and strategic behavior in a multiregional transboundary pollution dynamic game. Journal of Environmental Economics and Management (2017) http://dx.doi.org/10.1016/j.jeem.2017.08.001.

[12] C. Galusinski and M. Saad, On a degenerate parabolic system for compressible, immiscible, two-phase flows in porous media. Advances in Differential Equations 9 (2004) 1235-1278.

[13] W. Hamdi, F. Gamaoun, D.E. Pelster and M. Seffen, Nitrate sorption in an agricultural soil profile. Applied and Environmental Soil Science (2013) http://dx.doi.org/10.1155/2013/597824. 
[14] A. Haraux and F. Murat, Influence of a singular perturbation on the infimum of some functionals. Journal of Differential Equations 58 (1985) 43-75, http://dx.doi.org/10.1016/0022-0396(85)90022-1.

[15] P.I. Kogut and G.R. Leugering, Optimal Control Problems for Partial Differential Equations on Reticulated Domains. Approximation and Asymptotic Analysis. Systems and Control: Foundations and Applications, Birkhäuser (2011) http://dx.doi.org/10.1007/978-0-8176-8149-4.

[16] O. A. Ladyzhenskaya and N.N. Ural'tseva, Linear and Quasilinear Elliptic Equations. Mathematics in Sciences in Engineering, Academic Press, (1968).

[17] J. Lankoski and M. Ollikainen, Innovations in nonpoint source pollution policy. European perspectives. The Magazine of Food Farm Resource issues 28(3) (2013) 3rd Quarter.

[18] J.L. Lions and E.Magenes, Problèmes aux limites non homogènes (II), Annales de l'Institut Fourier 11 (1961) $137-178$.

[19] G. de Marsily, Quantitative Hydrogeology: Groundwater Hydrology for Engineers, Academic Press (1986).

[20] F. van der Ploeg and A. de Zeeuw, A differential game of international pollution control. Systems 8 Control Letters 17(6) (1991) 409-414, http://dx.doi.org/10.1016/0167-6911(91)90080-X.

[21] C. S. Rogers, Economic costs of conventional surface-water treatment: A case study of the Mcallen Northwest Facility. Master of Science Dissertation, Texas A\&M University, USA (2010).

[22] A. E. Scheidegger, The Physics of Flow through Porous Media. University of Toronto Press (1974)

[23] J. Simon, Compact sets in the space $L^{p}(0, T ; B)$, Annali di matematica pura ed applicata (IV) (1987) Vol. CXLVI, 65-96, https://doi.org/10.1007/BF01762360

[24] L.C. Tartar, Compensated compactness and applications to partial differential equations, in R. J. Knops, Ed., Research Notes in Mathematics, Nonlinear Analysis and Mechanics, Heriot-Watt Symposium, Vol. 4, Pitman Press, London, (1979).

[25] F.A. Williams, Combustion Theory: the fundamental Theory of chemically reacting Flow Systems, Benjamin-Cummings Pub. Co., Menlo Park, Calif., 2nd ed., (1985).

[26] Y. Yuan, D. Liang and H. Zhu, Optimal control of groundwater pollution combined with source abatement costs and taxes. Journal of Computational Science 20 (2017) 17-29, http://dx.doi.org/10.1016/j.jocs.2017.03.014.

[27] Commissariat général au développement durable : Les teneurs en nitrates augmentent dans les nappes phréatiques jusqu'en 2004 puis se stabilisent, Commissariat général au développement durable, Observations et Statistiques, numéro 16, mai 2013, from the www.statistiques.developpement-durable.gouv.fr 